\title{
Nonparametric Competitors to the Two-Way ANOVA
}

\author{
Larry E. Toothaker and De Newman \\ University of Oklahoma
}

Key words: two-way designs, ANOVA, nonparametric tests

\begin{abstract}
The ANOVA $F$ and several nonparametric competitors for two-way designs were compared for empirical $\alpha$ and power. Simulation of $2 \times 2,2 \times 4$, and $4 \times 4$ designs was done with cell sizes of 5 and 10 when sampling from normal, exponential, and mixed normal distributions. Conservatism of both $\alpha$ and power in the presence of other nonnull effects was seen in the tests due to Puri and Sen (1985) and, to a lesser degree, in the rank transform tests (Conover \& Iman, 1981). Tests by McSweeney (1967) and Hettmansperger (1984) had liberal $\alpha$ for some designs and distributions, especially for small $n$. The ANOVA $F$ suffers from conservative $\alpha$ and power for the mixed normal distribution, but it is generally recommended.
\end{abstract}

The applied researcher has often expressed concerns about meeting the assumptions of the parametric fixed effects two-way ANOVA. Given the linear model of $Y_{i j k}=\mu+\alpha_{j}+\beta_{k}+\alpha \beta_{j k}+e_{i j k}$, the assumptions on the error are that $e_{i j k}{ }_{i}^{d} \operatorname{NID}\left(0, \sigma_{e}^{2}\right)$ for each cell. These concerns about assumptions have been addressed in research on ANOVA, including development of alternatives to the ANOVA and examination of the ANOVA under assumption violations.

Nonparametric competitors to the two-way ANOVA relax the assumptions on $e_{i j k}$ by assuming that they are independent, identically distributed random variables from some continuous distribution. Nonparametrics for the twoway layout include rank tests by Puri and Sen (1985), rank transform tests by Conover and Iman (1981), and aligned rank tests. The latter area has grown out of a suggestion by Hodges and Lehmann (1962) and includes a test for interaction by Mehra and Sen (1969), tests for main effects in an additive model by Mehra and Sarangi (1967) and Sen (1968), tests for interaction in mixed models by Koch (1969) and Koch and Sen (1968), a test for interaction in the presence of main effects by McSweeney (1967), and linear-model-based tests of main effects and interaction by Hettmansperger (1984). Generally, nonparametric methods have gained attention due to their ability to analyze data that is questionable with respect to the normality assumption. These nonparametric competitors to the two-way ANOVA would be desirable alternatives for nonnormal data likely to be found in applied 
research (see Micceri, 1989) if they provide adequate control of $\alpha$ and power competitive to that of the $F$ tests in the ANOVA.

The purpose of this article is to present the results of Monte Carlo simulations of four general groups of statistical methods for two-way designs: (a) parametric two-way ANOVA, (b) Puri and Sen (1985) tests, (c) rank transform tests, and (d) aligned rank tests. The latter group includes tests for all three effects from McSweeney (1967), Hettmansperger (1984), and ANOVA $F$ tests on ranks aligned on all other effects possible.

\section{Rank Tests}

\section{Puri and Sen Tests}

The rank tests given by Puri and Sen (PS) (1985) for linear models can be adopted for tests on main effects and interaction in a two-way layout and can be presented as a function of the proportion of variability due to the desired effect (see Harwell, 1991; Harwell \& Serlin, 1990). Given $n$ observations per cell, $J$ rows, and $K$ columns, the steps to do these tests are as follows:

1. Rank all the data from one to total sample size, $N=n J K$.

2. Compute the sums of squares for rows, $\mathrm{SS}_{\mathrm{R}}$, columns, $\mathrm{SS}_{\mathrm{C}}$, interaction, $\mathrm{SS}_{\mathrm{RC}}$, and total, $\mathrm{SS}_{\mathrm{T}}$.

3. Compute the statistics $(N-1) \mathrm{SS}_{\mathrm{R}} / \mathrm{SS}_{\mathrm{T}},(N-1) \mathrm{SS}_{\mathrm{C}} / \mathrm{SS}_{\mathrm{T}}$, and $(N-1) \mathrm{SS}_{\mathrm{RC}} / \mathrm{SS}_{\mathrm{T}}$.

4. Refer the statistics to chi-square critical values with $d f_{\mathrm{R}}=J-1$, $d f_{\mathrm{c}}=K-1$, and $d f_{\mathrm{RC}}=(J-1)(K-1)$, respectively.

Steps 1 and 2 could be accomplished easily with any major statistical package such as SAS (1990), using PROC RANK to do the ranking and PROC ANOVA to compute the sums of squares. It should be noted that these procedures are considered large-sample tests, because the statistics are only asymptotically chi-square.

Other researchers developed equivalents of the PS rank tests, but Puri and Sen (1985) were the first to present them in a general linear model approach which has broader use than the two-way ANOVA. Reinach (1965) suggested that rank tests using an ANOVA approach would be confounded by the presence of other nonnull effects. Scheirer, Ray, and Hare (1976) gave statistics that are equivalent to the PS statistics for a two-way design and presented Monte Carlo results for small $n$ with a normal distribution. Scheirer et al. (1976) found that the statistics gave adequate control of Type I error rate for main effects and interaction tests, but they investigated only the completely null case. That is, the probability of Type I error was examined for each of the row, column, and interaction tests in the presence of null effects. For a normal distribution, Toothaker and Chang (1980) found that the Scheirer et al. statistics (PS tests) exhibited a dampening effect in the presence of any other nonnull effect. That is, the PS tests became more conservative as the 
magnitude of the other effects increased. The conservatism of the PS tests was found for both $\alpha$ and power and for both main effects and interaction tests. This conservatism was found even in the presence of one nonnull effect which would give power of only about .60. Also, Lemmer (1980) presented a statistic which is equivalent to PS tests, and found conservatism of $\alpha$ which became worse as magnitude or number of other effects increased.

Harwell (1991) presented results for the PS tests for small $n$ and found that the PS tests controlled $\alpha$ in the completely null case for five distributions: normal, double-exponential, approximate Cauchy, chi-square with $d f=8$, and chi-square with $d f=2$ (exponential). However, when one nonnull effect, which would give power of about .30 for $n=5$, is present, PS tests showed conservatism consistent with that found by Toothaker and Chang (1980). This conservatism of the PS tests was most severe for the chi-square distribution with $d f=2$. Power results showed "almost uniformly lower power values than its [PS test] competitors for all distributions" (p. 396), but Harwell (1991) concluded that there was no power decrement or dampening effect on power. Harwell did not observe the dampening effect on power in the PS statistics because he used only one degree of nonnullity for each sample size. However, evidence of the decrement in power is available from his data: when sampling from a normal distribution with one nonnull effect, for $n=$ 5 , power is .313 for interaction, but, when all effects are nonnull, power is .256 . Similarly, for the chi-square distribution with $d f=2$, the power decrement is from .552 to .319 . However, the dampening effect is not always present for all PS tests for all distributions. Harwell (1991) also noted a potential problem for PS tests in that the ranking process "can create a serious mismatch between effects in the model purported to underlie the original scores and effects in the ranks of the scores" (p. 384). More will be given on this problem when rank transform tests are discussed.

\section{Rank Transform Tests (RT)}

Tests known as rank transforms have been popularized by Conover and Iman (1976, 1981), Iman (1974), and Iman and Conover (1976). The steps to do RT tests in the two-way layout are as follows:

1. Rank all the data from one to total sample size, $N=n J K$.

2. Compute the sums of squares for rows, $\mathrm{SS}_{\mathrm{R}}$, columns, $\mathrm{SS}_{\mathrm{C}}$, interaction, $\mathrm{SS}_{\mathrm{RC}}$, and total, $\mathrm{SS}_{\mathrm{T}}$.

3. Compute the ANOVA $F$ statistics $F_{\mathrm{R}}=\mathrm{MS}_{\mathrm{R}} / \mathrm{MS}_{\mathrm{W}}, F_{\mathrm{C}}=\mathrm{MS}_{\mathrm{C}} / \mathrm{MS}_{\mathrm{W}}$, and $F_{\mathrm{RC}}=\mathrm{MS}_{\mathrm{RC}} / \mathrm{MS}_{\mathrm{w}}$.

4. Refer the statistics to $F$ critical values with $d f_{\mathrm{R}}=J-1, d f_{\mathrm{C}}=$ $K-1$, and $d f_{\mathrm{RC}}=(J-1)(K-1)$, in the numerators, respectively, and $d f_{\mathrm{W}}=J K(n-1)$ in the denominators.

Again, these steps could easily be accomplished with any major statistical package such as SAS, using PROC RANK to do the ranking and PROC 
ANOVA to compute the $F$ ratios. In fact, SAS (1990, p. 493) says that data can be submitted to PROC RANK and then fitted with an ANOVA model using the ANOVA or GLM procedures. It should be noted that RT procedures are considered large-sample tests, because the statistics are only asymptotically distributed as $F$.

In spite of much positive research on RT tests for two-way layouts (see Hora \& Conover, 1984; Hora \& Iman, 1988; Iman, 1974; Iman \& Conover, 1976; Iman, Hora, \& Conover, 1984; Thompson \& Ammann, 1989), recent results show RT tests lack control of $\alpha$ in the presence of other nonnull effects (see Sawilowsky, 1990). Fawcett and Salter (1984) found increasing conservatism as the degree of nonnullity of block effects increased in a RT test for treatments in an additive model randomized block design with $n=$ 1. In an additive model randomized block design with $n>1$, Groggel (1987) found that a RT test for treatment evidenced a decrease in power as block effects increased. Akritas (1990) showed that RT tests are confounded due to other nonnull effects. Thus RT tests should not be used to test for main effects in the presence of interaction, nor should RT tests be used to test interaction in the presence of main effects. The following all show that RT tests should not be used for two-way and higher ANOVA layouts: inflation of $\alpha$ for the $F$ for interaction in the presence of nonnull row and column effects in a two-way layout (Blair, Sawilowsky, \& Higgins, 1987), inflation of $\alpha$ for any effect and power for nonnull effects in a $2 \times 2 \times 2$ layout (Sawilowsky, Blair, \& Higgins, 1989), $\alpha$ and power properties being dependent on how the treatment effects were modeled in a $2 \times 2 \times 2$ layout (Sawilowsky, 1985), and inflation of $\alpha$ for a main effect in the presence of nonnull interaction (Lemmer, 1980). One exception to this warning is the 2 $\times 2$ layout, where the correct effects seem to be modeled by RT tests (Blair, Sawilowsky, \& Higgins, 1987) but where power is lower than the ANOVA on raw data for normal distributions (Sawilowsky, 1989). These results validate concerns expressed by Fligner (1981), "Rank transformation methods can provide quick solutions to many of these problems, but the resulting solutions may not be the best, or may not even be appropriate nonparametric methods." RT tests are included here to further examine these methods for two-way layouts.

\section{Aligned Rank Tests}

Given the problems encountered by PS tests and RT tests when other nonnull effects are present, one obvious solution would be to treat other effects as nuisance parameters and remove them from the scores before the ranking and analysis. Indeed, this is the principle on which aligned rank tests are based. For a two-way layout, the general steps to do aligned rank tests are as follows:

1. Estimate the effects on which the aligning is to be done. 
2. Subtract the estimated effects from the original scores (align the scores).

3. Rank the aligned scores in the total sample.

4. Compute the test statistic on the ranks of the aligned scores.

The actual effects of the model used to align scores and the eventual test statistic are the basis for the differences that exist in the several forms of aligned rank tests. Variations include different ways to estimate the desired effects-such as, means, trimmed means, medians-and different ways to form the test statistic - such as, using some function of sums of squares, which is asymptotically distributed as $\chi^{2}$, or an $F$ statistic, which asymptotically follows an $F$ distribution.

McSweeney (1967) developed a test (M test) for interaction using aligned ranks in the two-way layout with a nonadditive model. After the original scores have been aligned by subtracting estimates of both main effects and after the aligned scores have been ranked, the test statistic for interaction can be computed as $(N-1) \mathrm{SS}_{\mathrm{RC}} / \mathrm{SS}_{\mathrm{T}}$ and referred to a chi-square critical value with $d f_{\mathrm{RC}}=(J-1)(K-1)$. By analogy, the test for row (column) main effects can be done as follows: Align scores on the estimates of the column (row) and interaction effects; rank the aligned scores, and compute $(N-1) \mathrm{SS}_{\mathrm{R}} / \mathrm{SS}_{\mathrm{T}}$ (or $(N-1) \mathrm{SS}_{\mathrm{C}} / \mathrm{SS}_{\mathrm{T}}$ ), which is referred to a chi-square critical value with $d f_{\mathrm{R}}=J-1$ (or $d f_{\mathrm{C}}=K-1$ ).

Hettmansperger (1984) developed a linear model or regression-based system from which tests ( $\mathrm{H}$ tests) for a two-way layout could be identified. Harwell (1991) presents these as procedures using ranks on aligned scores, where the aligning is done on all effects in the model that are not being tested. For example, for the interaction test, after the original scores have been aligned by subtracting estimates of both main effects, obtain ranks of the aligned scores, $r_{i j k}$. Standardize the ranks to have mean zero and variance one by using

$$
r_{i j k}^{\prime}=\left(\frac{r_{i j k}}{N+1}-.5\right)(\sqrt{12}),
$$

and use $r_{i j k}^{\prime}$ to compute $\mathrm{SS}_{\mathrm{Rc}}$. Two different critical values were proposed by Hettmansperger (1984): a chi-square critical value with $d f_{\mathrm{RC}}=(J-1)(K-1)$ and a $\left(d f_{\mathrm{RC}}\right) F$ critical value with degrees of freedom $d f_{\mathrm{RC}}$ and $d f_{\mathrm{W}}$. Preference is given for the latter due to liberal simulation results (cited in Hettmansperger, 1984) when using the chi-square critical value. Similarly, the test for row (column) main effects can be done as follows: Align scores on the estimates of the column (row) and interaction effects; rank the aligned scores; standardize the ranks using Equation 1; use $r_{i j k}^{\prime}$ to compute $\mathrm{SS}_{\mathrm{R}}$ (or $\mathrm{SS}_{\mathrm{C}}$ ), which is referred to a chi-square critical value with $d f_{\mathrm{R}}=J-1\left(\right.$ or $\left.d f_{\mathrm{C}}=K-1\right)$. An alternate $F$-based critical value would be 
$\left(d f_{\mathrm{R}}\right) F$ with degrees of freedom $d f_{\mathrm{R}}$ and $d f_{\mathrm{W}}$ (or $\left(d f_{\mathrm{C}}\right) F$ with degrees of freedom $d f_{\mathrm{C}}$ and $d f_{\mathrm{w}}$ ). Note that three separate rankings must be done for McSweeney or Hettmansperger tests.

A final aligned rank test to be considered would be the ANOVA $F$ tests on ranks aligned on all other effects possible (RO tests). The aligning and ranking are the same as those shared by the McSweeney and Hettmansperger tests, but the test statistics differ. After aligning the raw data by subtracting estimates of both main effects and obtaining ranks of all of the aligned data, the statistic for interaction is the ANOVA $F$ on ranks, which is then referred to a $F$ critical value with $d f_{\mathrm{RC}}$ and $d f_{\mathrm{w}}$. Statistics for the main effects come from two additional rankings, where the data are first aligned by subtracting estimates of the other main effect and the interaction. Then the ANOVA $F_{\mathrm{R}}$ $\left(F_{\mathrm{C}}\right)$ on ranks is referred to a $F$ critical value with $d f_{\mathrm{R}}$ and $d f_{\mathrm{W}}\left(d f_{\mathrm{C}}\right.$ and $\left.d f_{\mathrm{W}}\right)$. These tests could be thought of as rank transform tests after aligning on all other possible effects.

In summary, the PS, RT, M, H, and RO tests for two-factor designs have in common the use of ranks, but they differ with respect to how the ranks are operationalized into a test statistic. The PS and RT tests do not align the observations before ranking, and the PS tests are functions of sums of squares, which are referred to chi-square critical values, while the RT tests compute $F$ s, which are referred to $F$ critical values. $\mathrm{M}, \mathrm{H}$, and $\mathrm{RO}$ all align on all other effects but compute functions of sums of squares referred to chi-square critical values, sums of squares of standardized ranks referred to $(d f) F$ critical values, and $F$ s which are referred to $F$ critical values, respectively.

\section{Method}

A computer program was written to perform simulations of the various tests, including the ANOVA $F$, for $2 \times 2,2 \times 4$, and $4 \times 4$ designs, for cell sizes of $n=5$ and $n=10$, and for normal, exponential, and mixed-normal distributions. Microsoft FORTRAN was used, and the program was run on a Tri-Star 386/33 with a math coprocessor.

Because of similar results for various levels of $\alpha$ in previous studies (see Harwell, 1991; Toothaker \& Chang, 1980), only nominal $\alpha$ of .05 was used. One null and two nonnull cases were examined for both main and interaction effects. Nonnull effects were chosen so as to give normal-theory power of approximately .85, called medium, and .99 , called large. Table 1 shows all cases obtained by combining main and interaction effects.

For the normal population, data were generated using the Box and Muller (1958) method, transforming independent unit uniform pseudorandom numbers from a procedure by Chen (1971). For the exponential population, data were generated by a method reported by Lehmann and Bailey (1968): $f(t)=$ $p \mathrm{e}^{-p t}$, with $p=1, \mathrm{E}(t)=1 / p=1$, and $\operatorname{var}(t)=1 / p^{2}=1$. Pseudorandom exponential variables were generated by multiplying -1 by the natural loga- 
TABLE 1

Cases of main and interaction effects

\begin{tabular}{|c|c|c|c|}
\hline Case & A main effect & B main effect & Interaction effect \\
\hline 1 & Null $^{\mathrm{a}}$ & Null & Null \\
\hline 2 & Null & Null & Medium $^{b}$ \\
\hline 3 & Null & Null & $\operatorname{Large}^{\mathfrak{c}}$ \\
\hline 4 & Null & Medium & Null \\
\hline 5 & Null & Medium & Medium \\
\hline 6 & Null & Medium & Large \\
\hline 7 & Null & Large & Null \\
\hline 8 & Null & Large & Medium \\
\hline 9 & Null & Large & Large \\
\hline 10 & Medium & Null & Null \\
\hline 11 & Medium & Null & Medium \\
\hline 12 & Medium & Null & Large \\
\hline 13 & Medium & Medium & Null \\
\hline 14 & Medium & Medium & Medium \\
\hline 15 & Medium & Medium & Large \\
\hline 16 & Medium & Large & Null \\
\hline 17 & Medium & Large & Medium \\
\hline 18 & Medium & Large & Large \\
\hline 19 & Large & Null & Null \\
\hline 20 & Large & Null & Medium \\
\hline 21 & Large & Null & Large \\
\hline 22 & Large & Medium & Null \\
\hline 23 & Large & Medium & Medium \\
\hline 24 & Large & Medium & Large \\
\hline 25 & Large & Large & Null \\
\hline 26 & Large & Large & Medium \\
\hline 27 & Large & Large & Large \\
\hline
\end{tabular}

${ }^{a}$ Null $=$ all effects are equal to zero, ${ }^{\mathrm{b}}$ Medium $=$ effects are selected so that power will be about .85 for the normal distribution, ${ }^{c}$ Large $=$ effects are selected so that power will be about .99 for the normal distribution.

rithm of unit uniform pseudorandom numbers (Chen, 1971). The exponential variates were then scaled to have means determined by the null or nonnull conditions and variance of one. For the mixed normal population, data were generated by generating $95 \%$ of the scores from $\mathrm{N}(0,1)$ and $5 \%$ of the scores from $N(22,100)$ using the Box and Muller (1958) and Chen (1971) methods. These variates, with mean of 1.1 and variance of 8.375 , were then scaled to have the mean determined by the null or nonnull conditions and variance of one. For any tests requiring aligning, estimates of aligned effects were computed using appropriate sample means. 
Empirical $\alpha$ and power were obtained over 5,000 replications, but the main effect information was collapsed for those cases where the degrees of freedom and the process of ranking/aligning were the same for both main effects. Thus, the values in the tables for those cases are averages of two proportions obtained over 5,000 replications. Note that all 27 cases in Table 1 were examined for the same 5,000 replications, thus controlling sampling variability between cases for each design, sample size, and distribution combination.

\section{Results}

Because the sampling variability of the empirical estimates was small, $\sigma_{p}$ $=.0030822$ for $\alpha=.05$ and 5,000 replications, arbitrary standards were used to decide when a statistic was evidencing lack of $\alpha$ robustness. Values of empirical $\alpha$ that deviated \pm .01 from .05 were called slightly liberal or slightly conservative, and values that deviated \pm .02 were called liberal or conservative. Thus, a value of empirical $\alpha$ of .057 was more than 2 standard deviations from .05 but was deemed not indicative of lack of robustness. Power robustness was determined by comparison of values for the various statistical procedures-that is, a method was called conservative if it showed power which was substantially less than that for the other methods.

\section{Normal Distribution}

Empirical $\alpha$ results for $n=5$ showed (see Table 2) M tests to be slightly liberal or liberal for all designs. $\mathrm{H}$ tests were slightly liberal or liberal for the main effect tests in the larger two designs and for the interaction test in the smaller two designs. $F$ tests were not liberal or conservative, and RO tests were slightly liberal only for interaction in the $2 \times 2$ design. PS tests showed conservatism in the presence of other nonnull effects which worsened as the number of nonnull effects or degree of nonnullity increased. For example, in the $2 \times 2$ design, in the presence of the other main effect with medium power (Cases 4 and 10), empirical $\alpha$ for the PS main effect test was .0177 , but, in the presence of the other two effects with large power (Cases 9 and 21), the main effect had an empirical $\alpha$ of .0002 . Similar results were seen for the PS interaction test. RT tests showed conservatism in the $2 \times 2$ design only if both other effects were nonnull. Power results showed PS tests with conservatism in the presence of other nonnull effects that dramatically worsened as the number of nonnull effects or degree of nonnullity increased. RT tests evidenced somewhat less power conservatism than that shown by PS, with large power loss only for the $2 \times 2$ design if both other effects were nonnull. Note that the slight power advantage of $H$ and $M$ tests is probably due to liberal $\alpha$.

For $n=10$ and empirical $\alpha$ results (see Table 3), the $M$ and $\mathrm{H}$ tests were slightly liberal for only the main effect test in the $4 \times 4$ design. Other tests showed results similar to those found for $n=5$. Power results were similar 
TABLE 2

Normal distribution, $\mathrm{n}=5$

\begin{tabular}{|c|c|c|c|c|c|c|c|c|c|}
\hline \multirow[b]{2}{*}{ Stat } & \multirow[b]{2}{*}{ Case } & \multirow[b]{2}{*}{$2 \times 2$} & \multicolumn{2}{|c|}{ Main effect } & \multirow[b]{2}{*}{$4 \times 4$} & \multirow[b]{2}{*}{ Case } & \multicolumn{3}{|c|}{ Interaction } \\
\hline & & & $2 \times 4 d f=1$ & $2 \times 4 d f=3$ & & & $2 \times 2$ & $2 \times 4$ & $4 \times 4$ \\
\hline \multicolumn{10}{|c|}{ Empirical Alpha } \\
\hline $\mathrm{F}$ & Null & $.0565+$ & .0528 & .0518 & $.0494+$ & Null & .0556 & .0524 & .0506 \\
\hline RO & Null & $.0584+$ & .0516 & .0534 & $.0499+$ & Null & .0602 & .0526 & .0518 \\
\hline $\mathrm{H}$ & Null & $.0589+$ & .0648 & .0602 & $.0756+$ & Null & .0604 & .0616 & .0588 \\
\hline M & Null & $.0717+$ & .0694 & .0664 & $.0784+$ & Null & .0732 & .0674 & .0630 \\
\hline RT & 1 & $.0551+$ & .0506 & .0536 & $.0500+$ & 1 & .0570 & .0538 & .0508 \\
\hline PS & 1 & $.0553+$ & .0488 & .0480 & $.0472+$ & 1 & .0588 & .0460 & .0420 \\
\hline RT & 2 & $.0531+$ & .0498 & .0524 & $.0505+$ & 4 & .0520 & .0566 & .0508 \\
\hline PS & 2 & $.0181+$ & .0218 & .0124 & $.0199+$ & 4 & .0184 & .0136 & .0150 \\
\hline RT & 3 & $.0527+$ & .0512 & .0502 & $.0506+$ & 7 & .0546 & .0532 & .0534 \\
\hline PS & 3 & $.0048+$ & .0104 & .0036 & $.0089+$ & 7 & .0064 & .0038 & .0064 \\
\hline RT & $4 \& 10$ & $.0534+$ & .0518 & .0522 & $.0487+$ & 10 & .0568 & .0542 & .0556 \\
\hline PS & $4 \& 10$ & $.0177+$ & .0232 & .0184 & $.0265+$ & 10 & .0198 & .0196 & .0170 \\
\hline RT & $5 \& 11$ & $.0211+$ & .0522 & .0450 & $.0490+$ & 13 & .0250 & .0538 & .0490 \\
\hline PS & $5 \& 11$ & $.0056+$ & .0088 & .0050 & $.0123+$ & 13 & .0076 & .0058 & .0046 \\
\hline RT & $6 \& 12$ & $.0117+$ & .0532 & .0428 & $.0492+$ & 16 & .0140 & .0516 & .0522 \\
\hline PS & $6 \& 12$ & $.0019+$ & .0038 & .0016 & $.0055+$ & 16 & .0022 & .0020 & .0020 \\
\hline RT & $7 \& 19$ & $.0534+$ & .0490 & .0520 & $.0480+$ & 19 & .0558 & .0530 & .0554 \\
\hline PS & $7 \& 19$ & $.0063+$ & .0104 & .0070 & $.0164+$ & 19 & .0056 & .0090 & .0076 \\
\hline
\end{tabular}




\begin{tabular}{|c|c|c|c|c|c|c|c|c|c|}
\hline \multirow[b]{2}{*}{ Stat } & \multirow[b]{2}{*}{ Case } & \multirow[b]{2}{*}{$2 \times 2$} & \multicolumn{2}{|c|}{ Main effect } & \multirow[b]{2}{*}{$4 \times 4$} & \multirow[b]{2}{*}{ Case } & \multicolumn{3}{|c|}{ Interaction } \\
\hline & & & $2 \times 4 d f=1$ & $2 \times 4 d f=3$ & & & $2 \times 2$ & $2 \times 4$ & $4 \times 4$ \\
\hline RT & $8 \& 20$ & $.0130+$ & .0492 & .0444 & $.0485+$ & 22 & .0148 & .0520 & .0504 \\
\hline PS & $8 \& 20$ & $.0016+$ & .0038 & .0026 & $.0081+$ & 22 & .0018 & .0016 & .0022 \\
\hline RT & $9 \& 21$ & $.0043+$ & .0474 & .0404 & $.0490+$ & 25 & .0040 & .0518 & .0512 \\
\hline PS & $9 \& 21$ & $.0002+$ & .0026 & .0010 & $.0044+$ & 25 & .0002 & .0006 & .0006 \\
\hline \multicolumn{10}{|c|}{ Empirical power (medium) } \\
\hline $\mathrm{F}$ & Med. & $.8513+$ & .8488 & .8618 & $.8525+$ & Med. & .8506 & .8500 & .8412 \\
\hline RO & Med. & $.8442+$ & .8340 & .8450 & $.8363+$ & Med. & .8478 & .8442 & .8340 \\
\hline $\mathrm{H}$ & Med. & $.8448+$ & .8616 & .8598 & $.8820+$ & Med. & .8488 & .8594 & .8444 \\
\hline $\mathbf{M}$ & Med. & $.8656+$ & .8690 & .8688 & $.8871+$ & Med. & .8694 & .8678 & .8554 \\
\hline RT & $10 \& 4$ & $.8366+$ & .8332 & .8432 & $.8357+$ & 2 & .8380 & .8404 & .8292 \\
\hline PS & $10 \& 4$ & $.8418+$ & .8344 & .8284 & $.8300+$ & 2 & .8426 & .8290 & .8078 \\
\hline RT & $11 \& 5$ & $.8221+$ & .8254 & .8310 & $.8282+$ & 5 & .8222 & .8304 & .8234 \\
\hline PS & $11 \& 5$ & $.6781+$ & .7242 & .6592 & $.7203+$ & 5 & .6716 & .6584 & .6762 \\
\hline RT & $12 \& 6$ & $.8168+$ & .8240 & .8260 & $.8272+$ & 8 & .8140 & .8242 & .8220 \\
\hline PS & $12 \& 6$ & $.5026+$ & .6190 & .4900 & $.6180+$ & 8 & .4958 & .4972 & .5686 \\
\hline RT & 13 & $.8210+$ & .8272 & .8346 & $.8336+$ & 11 & .8208 & .8362 & .8220 \\
\hline PS & 13 & $.6764+$ & .7212 & .7206 & $.7630+$ & 11 & .6744 & .7176 & .6766 \\
\hline RT & 14 & $.6687+$ & .8076 & .8154 & $.8277+$ & 14 & .6606 & .8194 & .8180 \\
\hline PS & 14 & $.4373+$ & .6114 & .5408 & $.6432+$ & 14 & .4288 & .5414 & .5428 \\
\hline
\end{tabular}


TABLE 2 (continued)

\begin{tabular}{|c|c|c|c|c|c|c|c|c|c|}
\hline \multirow[b]{2}{*}{ Stat } & \multirow[b]{2}{*}{ Case } & \multirow[b]{2}{*}{$2 \times 2$} & \multicolumn{2}{|c|}{ Main effect } & \multirow[b]{2}{*}{$4 \times 4$} & \multirow[b]{2}{*}{ Case } & \multicolumn{3}{|c|}{ Interaction } \\
\hline & & & $2 \times 4 d f=1$ & $2 \times 4 d f=3$ & & & $2 \times 2$ & $2 \times 4$ & $4 \times 4$ \\
\hline RT & 15 & $.5464+$ & .7968 & .8076 & $.8243+$ & 17 & .5450 & .8128 & .8170 \\
\hline PS & 15 & $.2564+$ & .5046 & .3858 & $.5371+$ & 17 & .2510 & .3960 & .4372 \\
\hline RT & $16 \& 22$ & $.8149+$ & .8276 & .8314 & $.8310+$ & 20 & .8144 & .8324 & .8196 \\
\hline PS & $16 \& 22$ & $.5012+$ & .6226 & .5832 & $.6957+$ & 20 & .4990 & .5792 & .5550 \\
\hline RT & $17 \& 23$ & $.5556+$ & .8070 & .8024 & $.8240+$ & 23 & .5436 & .8106 & .8168 \\
\hline PS & $17 \& 23$ & $.2540+$ & .5094 & .4226 & $.5763+$ & 23 & .2516 & .4128 & .4398 \\
\hline RT & $18 \& 24$ & $.3480+$ & .7932 & .7916 & $.8202+$ & 26 & .3398 & .8008 & .8116 \\
\hline PS & $18 \& 24$ & $.1258+$ & .4112 & .2922 & $.4740+$ & 26 & .1202 & .2908 & .3458 \\
\hline \multicolumn{10}{|c|}{ Empirical power (large) } \\
\hline $\mathrm{F}$ & Lar. & $.9893+$ & .9884 & .9896 & $.9875+$ & Lar. & .9914 & .9878 & .9900 \\
\hline RO & Lar. & $.9860+$ & .9854 & .9884 & $.9836+$ & Lar. & .9878 & .9868 & .9894 \\
\hline $\mathrm{H}$ & Lar. & $.9862+$ & .9888 & .9904 & $.9904+$ & Lar. & .9880 & .9892 & .9906 \\
\hline $\mathbf{M}$ & Lar. & $.9897+$ & .9892 & .9906 & $.9910+$ & Lar. & .9912 & .9902 & .9914 \\
\hline RT & $19 \& 7$ & $.9864+$ & .9856 & .9870 & $.9835+$ & 3 & .9878 & .9862 & .9892 \\
\hline PS & $19 \& 7$ & $.9867+$ & .9860 & .9846 & $.9834+$ & 3 & .9886 & .9838 & .9864 \\
\hline RT & $20 \& 8$ & $.9827+$ & .9842 & .9870 & $.9825+$ & 6 & .9854 & .9838 & .9878 \\
\hline PS & $20 \& 8$ & $.9500+$ & .9628 & .9436 & $.9577+$ & 6 & .9528 & .9458 & .9646 \\
\hline RT & $21 \& 9$ & $.9810+$ & .9832 & .9876 & $.9824+$ & 9 & .9842 & .9830 & .9880 \\
\hline PS & $21 \& 9$ & $.8823+$ & .9350 & .8786 & $.9271+$ & 9 & .8738 & .8852 & .9310 \\
\hline
\end{tabular}


TABLE 2 (continued)

\begin{tabular}{|c|c|c|c|c|c|c|c|c|c|}
\hline \multirow[b]{2}{*}{ Stat } & \multirow[b]{2}{*}{ Case } & \multirow[b]{2}{*}{$2 \times 2$} & \multicolumn{2}{|c|}{ Main effect } & \multirow[b]{2}{*}{$4 \times 4$} & \multirow[b]{2}{*}{ Case } & \multicolumn{3}{|c|}{ Interaction } \\
\hline & & & $2 \times 4 d f=1$ & $2 \times 4 d f=3$ & & & $2 \times 2$ & $2 \times 4$ & $4 \times 4$ \\
\hline $\mathrm{RT}$ & $22 \& 16$ & $.9827+$ & .9840 & .9856 & $.9827+$ & 12 & .9840 & .9840 & .9884 \\
\hline PS & $22 \& 16$ & $.9495+$ & .9700 & .9626 & $.9687+$ & 12 & .9570 & .9598 & .9644 \\
\hline $\mathrm{RT}$ & $23 \& 17$ & $.9437+$ & .9828 & .9856 & $.9811+$ & 15 & .9428 & .9802 & .9870 \\
\hline PS & $23 \& 17$ & $.8333+$ & .9310 & .9070 & $.9364+$ & 15 & .8284 & .9028 & .9236 \\
\hline RT & $24 \& 18$ & $.8895+$ & .9810 & .9830 & $.9805+$ & 18 & .8894 & .9792 & .9858 \\
\hline PS & $24 \& 18$ & $.6738+$ & .8934 & .8222 & $.8924+$ & 18 & .6700 & .8244 & .8716 \\
\hline RT & 25 & $.9822+$ & .9850 & .9850 & $.9817+$ & 21 & .9830 & .9834 & .9868 \\
\hline PS & 25 & $.8825+$ & .9406 & .9222 & $.9525+$ & 21 & .8824 & .9166 & .9314 \\
\hline $\mathrm{RT}$ & 26 & $.8931+$ & .9816 & .9818 & $.9800+$ & 24 & .8886 & .9784 & .9856 \\
\hline PS & 26 & $.6737+$ & .8978 & .8386 & $.9108+$ & 24 & .6666 & .8326 & .8722 \\
\hline RT & 27 & $.7548+$ & .9796 & .9790 & $.9797+$ & 27 & .7488 & .9754 & .9846 \\
\hline PS & 27 & $.4787+$ & .8460 & .7408 & $.8599+$ & 27 & .4670 & .7400 & .8082 \\
\hline
\end{tabular}

+Entry is averaged over two main effects. 
TABLE 3

Normal distribution, $\mathrm{n}=10$

\begin{tabular}{|c|c|c|c|c|c|c|c|c|c|}
\hline \multirow[b]{2}{*}{ Stat } & \multirow[b]{2}{*}{ Case } & \multirow[b]{2}{*}{$2 \times 2$} & \multicolumn{2}{|c|}{ Main effect } & \multirow[b]{2}{*}{$4 \times 4$} & \multirow[b]{2}{*}{ Case } & \multicolumn{3}{|c|}{ Interaction } \\
\hline & & & $2 \times 4 d f=1$ & $2 \times 4 d f=3$ & & & $2 \times 2$ & $2 \times 4$ & $4 \times 4$ \\
\hline \multicolumn{10}{|c|}{ Empirical $\alpha$} \\
\hline $\mathrm{F}$ & Null & $.0500+$ & .0490 & .0492 & $.0506+$ & Null & .0540 & .0474 & .0490 \\
\hline RO & Null & $.0519+$ & .0498 & .0484 & $.0500+$ & Null & .0554 & .0462 & .0482 \\
\hline $\mathrm{H}$ & Null & $.0519+$ & .0562 & .0510 & $.0616+$ & Null & .0554 & .0508 & .0524 \\
\hline $\mathbf{M}$ & Null & $.0552+$ & .0574 & .0536 & $.0633+$ & Null & .0592 & .0546 & .0544 \\
\hline RT & 1 & $.0508+$ & .0524 & .0510 & $.0515+$ & 1 & .0540 & .0490 & .0492 \\
\hline PS & 1 & $.0495+$ & .0516 & .0480 & $.0497+$ & 1 & .0528 & .0448 & .0456 \\
\hline RT & 2 & $.0506+$ & .0512 & .0514 & $.0523+$ & 4 & .0552 & .0488 & .0504 \\
\hline PS & 2 & $.0295+$ & .0354 & .0298 & $.0304+$ & 4 & .0326 & .0280 & .0326 \\
\hline RT & 3 & $.0513+$ & .0506 & .0526 & $.0520+$ & 7 & .0570 & .0506 & .0476 \\
\hline PS & 3 & $.0166+$ & .0258 & .0188 & $.0216+$ & 7 & .0162 & .0174 & .0216 \\
\hline $\mathrm{RT}$ & $4 \& 10$ & $.0504+$ & .0502 & .0512 & $.0513+$ & 10 & .0520 & .0496 & .0518 \\
\hline PS & $4 \& 10$ & $.0268+$ & .0324 & .0346 & $.0358+$ & 10 & .0288 & .0328 & .0302 \\
\hline RT & $5 \& 11$ & $.0412+$ & .0480 & .0492 & $.0503+$ & 13 & .0412 & .0486 & .0522 \\
\hline PS & $5 \& 11$ & $.0156+$ & .0230 & .0210 & $.0232+$ & 13 & .0162 & .0182 & .0220 \\
\hline RT & $6 \& 12$ & $.0364+$ & .0476 & .0474 & $.0511+$ & 16 & .0370 & .0474 & .0524 \\
\hline PS & $6 \& 12$ & $.0071+$ & .0174 & .0126 & $.0174+$ & 16 & .0096 & .0120 & .0148 \\
\hline RT & $7 \& 19$ & $.0479+$ & .0504 & .0502 & $.0505+$ & 19 & .0520 & .0486 & .0514 \\
\hline PS & $7 \& 19$ & $.0153+$ & .0242 & .0164 & $.0273+$ & 19 & .0158 & .0144 & .0206 \\
\hline
\end{tabular}


TABLE 3 (continued)

\begin{tabular}{|c|c|c|c|c|c|c|c|c|c|}
\hline \multirow[b]{2}{*}{ Stat } & \multirow[b]{2}{*}{ Case } & \multirow[b]{2}{*}{$2 \times 2$} & \multicolumn{2}{|c|}{ Main effect } & \multirow[b]{2}{*}{$4 \times 4$} & \multirow[b]{2}{*}{ Case } & \multicolumn{3}{|c|}{ Interaction } \\
\hline & & & $2 \times 4 d f=1$ & $2 \times 4 d f=3$ & & & $2 \times 2$ & $2 \times 4$ & $4 \times 4$ \\
\hline RT & $8 \& 20$ & $.0348+$ & .0458 & .0466 & $.0507+$ & 22 & .0382 & .0466 & .0530 \\
\hline PS & $8 \& 20$ & $.0080+$ & .0180 & .0092 & $.0176+$ & 22 & .0092 & .0088 & .0134 \\
\hline RT & $9 \& 21$ & $.0266+$ & .0470 & .0454 & $.0508+$ & 25 & .0272 & .0466 & .0522 \\
\hline PS & $9 \& 21$ & $.0034+$ & .0104 & .0052 & $.0138+$ & 25 & .0036 & .0048 & .0084 \\
\hline \multicolumn{10}{|c|}{ Empirical power (medium) } \\
\hline $\mathrm{F}$ & Med. & $.8514+$ & .8432 & .8488 & $.8238+$ & Med. & .8480 & .8432 & .8608 \\
\hline RO & Med. & $.8364+$ & .8324 & .8326 & $.8094+$ & Med. & .8354 & .8294 & .8494 \\
\hline $\mathrm{H}$ & Med. & $.8364+$ & .8474 & .8428 & $.8318+$ & Med. & .8354 & .8362 & .8568 \\
\hline M & Med. & $.8440+$ & .8498 & .8496 & $.8342+$ & Med. & .8424 & .8414 & .8606 \\
\hline RT & $10 \& 4$ & $.8339+$ & .8284 & .8344 & $.8061+$ & 2 & .8314 & .8272 & .8468 \\
\hline PS & $10 \& 4$ & $.8283+$ & .8294 & .8272 & $.8035+$ & 2 & .8294 & .8200 & .8404 \\
\hline RT & $11 \& 5$ & $.8309+$ & .8286 & .8312 & $.8045+$ & 5 & .8258 & .8236 & .8474 \\
\hline PS & $11 \& 5$ & $.7624+$ & .7898 & .7556 & $.7505+$ & 5 & .7576 & .7544 & .7988 \\
\hline RT & $12 \& 6$ & $.8266+$ & .8276 & .8290 & $.8037+$ & 8 & .8228 & .8244 & .8444 \\
\hline PS & $12 \& 6$ & $.6816+$ & .7456 & .6824 & $.7019+$ & 8 & .6776 & .6858 & .7490 \\
\hline RT & 13 & $.8250+$ & .8282 & .8302 & $.8136+$ & 11 & .8270 & .8264 & .8432 \\
\hline PS & 13 & $.7605+$ & .7906 & .7750 & $.7684+$ & 11 & .7598 & .7764 & .7942 \\
\hline RT & 14 & $.7999+$ & .8258 & .8268 & $.7911+$ & 14 & .7938 & .8180 & .8386 \\
\hline PS & 14 & $.6555+$ & .7392 & 6994 & $.7045+$ & 14 & .6558 & .6982 & .7446 \\
\hline
\end{tabular}


TABLE 3 (continued)

\begin{tabular}{|c|c|c|c|c|c|c|c|c|c|}
\hline \multirow[b]{2}{*}{ Stat } & \multirow[b]{2}{*}{ Case } & \multirow[b]{2}{*}{$2 \times 2$} & \multicolumn{2}{|c|}{ Main effect } & \multirow[b]{2}{*}{$4 \times 4$} & \multirow[b]{2}{*}{ Case } & \multicolumn{3}{|c|}{ Interaction } \\
\hline & & & $2 \times 4 d f=1$ & $2 \times 4 d f=3$ & & & $2 \times 2$ & $2 \times 4$ & $4 \times 4$ \\
\hline RT & 15 & $.7697+$ & .8222 & .8206 & $.7873+$ & 17 & .7676 & .8158 & .8382 \\
\hline PS & 15 & $.5508+$ & .6956 & .6234 & $.6523+$ & 17 & .5368 & .6270 & .6878 \\
\hline RT & $16 \& 22$ & $.8210+$ & .8254 & .8284 & $.8010+$ & 20 & .8250 & .8228 & .8428 \\
\hline PS & $16 \& 22$ & $.6802+$ & .7480 & .6696 & $.7350+$ & 20 & .6802 & .6714 & .7434 \\
\hline RT & $17 \& 23$ & $.7659+$ & .8218 & .8188 & $.7863+$ & 23 & .7702 & .8136 & .8336 \\
\hline PS & $17 \& 23$ & $.5455+$ & .6976 & .5890 & $.6634+$ & 23 & .5344 & .5886 & .6872 \\
\hline RT & $18 \& 24$ & $.7074+$ & .8162 & .8144 & $.7796+$ & 26 & .7090 & .8090 & .8312 \\
\hline PS & $18 \& 24$ & $.4182+$ & .6504 & .5170 & $.6046+$ & 26 & .4064 & .5162 & .6242 \\
\hline \multicolumn{10}{|c|}{ Empirical power (large) } \\
\hline $\mathrm{F}$ & Lar. & $.9898+$ & .9994 & .9880 & $.9877+$ & Lar. & .9896 & .9916 & .9928 \\
\hline RO & Lar. & $.9874+$ & .9992 & .9854 & $.9858+$ & Lar. & .9856 & .9884 & .9908 \\
\hline $\mathbf{H}$ & Lar. & $.9874+$ & .9996 & .9870 & $.9887+$ & Lar. & .9856 & .9890 & .9910 \\
\hline $\mathbf{M}$ & Lar. & $.9878+$ & .9996 & .9876 & $.9890+$ & Lar. & .9864 & .9896 & .9912 \\
\hline RT & $19 \& 7$ & $.9857+$ & .9992 & .9850 & $.9851+$ & 3 & .9856 & .9866 & .9906 \\
\hline PS & $19 \& 7$ & $.9855+$ & .9992 & .9850 & $.9848+$ & 3 & .9854 & .9856 & .9894 \\
\hline RT & $20 \& 8$ & $.9853+$ & .9990 & .9854 & $.9838+$ & 6 & .9838 & .9862 & .9894 \\
\hline PS & $20 \& 8$ & $.9743+$ & .9980 & .9698 & $.9746+$ & 6 & .9734 & .9728 & .9840 \\
\hline RT & $21 \& 9$ & $.9850+$ & .9992 & .9844 & $.9831+$ & 9 & .9828 & .9876 & .9896 \\
\hline PS & $21 \& 9$ & $.9535+$ & .9968 & .9542 & $.9645+$ & 9 & .9496 & .9556 & .9750 \\
\hline
\end{tabular}


TABLE 3 (continued)

\begin{tabular}{|c|c|c|c|c|c|c|c|c|c|}
\hline \multirow[b]{2}{*}{ Stat } & \multirow[b]{2}{*}{ Case } & \multirow[b]{2}{*}{$2 \times 2$} & \multicolumn{2}{|c|}{ Main effect } & \multirow[b]{2}{*}{$4 \times 4$} & \multirow[b]{2}{*}{ Case } & \multicolumn{3}{|c|}{ Interaction } \\
\hline & & & $2 \times 4 d f=1$ & $2 \times 4 d f=3$ & & & $2 \times 2$ & $2 \times 4$ & $4 \times 4$ \\
\hline RT & $22 \& 16$ & $.9859+$ & .9988 & .9838 & $.9848+$ & 12 & .9854 & .9862 & .9904 \\
\hline PS & $22 \& 16$ & $.9730+$ & .9984 & .9742 & $.9791+$ & 12 & .9724 & .9766 & .9844 \\
\hline RT & $23 \& 17$ & $.9800+$ & .9988 & .9834 & $.9825+$ & 15 & .9772 & .9858 & .9896 \\
\hline PS & $23 \& 17$ & $.9474+$ & .9970 & .9570 & $.9656+$ & 15 & .9394 & .9612 & .9732 \\
\hline RT & $24 \& 18$ & $.9746+$ & .9984 & .9828 & $.9807+$ & 18 & .9708 & .9848 & .9882 \\
\hline PS & $24 \& 18$ & $.9012+$ & .9942 & .9348 & $.9530+$ & 18 & .8980 & .9406 & .9628 \\
\hline RT & 25 & $.9845+$ & .9990 & .9830 & $.9851+$ & 21 & .9842 & .9860 & .9902 \\
\hline PS & 25 & $.9529+$ & .9966 & .9520 & $.9729+$ & 21 & .9488 & .9540 & .9748 \\
\hline RT & 26 & $.9746+$ & .9988 & .9826 & $.9814+$ & 24 & .9736 & .9844 & .9886 \\
\hline PS & 26 & $.9016+$ & .9952 & .9254 & $.9555+$ & 24 & .9006 & .9248 & .9616 \\
\hline RT & 27 & $.9597+$ & .9988 & .9812 & $.9796+$ & 27 & .9548 & .9838 & .9874 \\
\hline PS & 27 & $.8175+$ & .9928 & .8948 & $.9405+$ & 27 & .8258 & .8978 & .9464 \\
\hline
\end{tabular}

+Entry is averaged over two main effects. 
to those found for $n=5$, except that the power loss of PS and RT tests generally was not as severe. The empirical $\alpha$ values for the rank tests might differ from .05 because all the rank tests in this study are large-sample tests and only asymptotically distributed as $\chi^{2}$ or $F$. For the normal distribution (see Tables 2 and 3), the empirical $\alpha$ values for most rank tests are closer to .05 for $N=10$ than for $N=5$.

\section{Exponential Distribution}

For $n=5$ and empirical $\alpha$ results (see Table 4), the M tests were liberal or slightly liberal except for the $d f=3$ main effect in the $2 \times 4$ design (empirical $\alpha=.0594$ ). $\mathrm{H}$ tests were slightly liberal or liberal except for main effect and interaction tests in the $2 \times 2$ design, the $d f=3$ main effect test in the $2 \times 4$ design, and the interaction test in the $4 \times 4$ design. $F$ tests were not liberal or conservative, and RO tests were slightly liberal only for tests in the $4 \times 4$ design. PS tests showed the same pattern that occurred for the normal distribution: conservatism in the presence of other nonnull effects that worsened as the number of nonnull effects or degree of nonnullity increased. RT tests usually showed the same pattern that occurred for the normal distribution: conservatism in the $2 \times 2$ design only if both other effects were nonnull. Exceptions to this conservatism were slightly liberal interaction tests for the $4 \times 4$ design, if either main effect was large, and for the $2 \times 4$ design, if both main effects were large. Some power results were similar to those for the normal distribution: PS tests with conservatism in the presence of other nonnull effects, RT tests with somewhat less power conservatism than that shown by PS tests, and large power loss only for the $2 \times 2$ design if both other effects were nonnull. Power values for $F$ tests are lower than those of RO tests by about $5 \%-10 \%$ in those cases where RO tests had acceptable $\alpha$. Note that at least some of the power advantage of $\mathrm{H}$ and $M$ tests is probably due to slightly liberal or liberal $\alpha$.

For $n=10$ and empirical $\alpha$ results (see Table 5), RO tests were not liberal, and the $\mathrm{M}$ and $\mathrm{H}$ tests were slightly liberal for only the main effect test in the $4 \times 4$ design. Other tests showed results similar to those found for $n=$ 5. Power results were similar to those found for $n=5$, except that the power loss of PS and RT tests generally was not as severe and that the power decrement of $F$, compared to RO, ranged as high as $13 \%$.

\section{Mixed Normal Distribution}

For $n=5$ and empirical $\alpha$ results (see Table 6), $F$ was always conservative. $\mathrm{H}$ and $\mathrm{M}$ gave acceptable $\alpha$ only for the interaction test in the $2 \times 4$ design. Conservatism was evidenced for $\mathrm{H}$ and $\mathrm{M}$ in main effect and interaction tests for the $2 \times 2$ design, the $d f=3$ test in the $2 \times 4$ design, and the interaction test in the $4 \times 4$ design. However, the $d f=1$ main effect test in the $2 \times 4$ design and main effect test in the $4 \times 4$ design were liberal for $\mathrm{H}$ and $\mathrm{M}$. 
TABLE 4

Exponential distribution, $\mathrm{n}=5$

\begin{tabular}{|c|c|c|c|c|c|c|c|c|c|}
\hline \multirow[b]{2}{*}{ Stat } & \multirow[b]{2}{*}{ Case } & \multirow[b]{2}{*}{$2 \times 2$} & \multicolumn{2}{|c|}{ Main effect } & \multirow[b]{2}{*}{$4 \times 4$} & \multirow[b]{2}{*}{ Case } & \multicolumn{3}{|c|}{ Interaction } \\
\hline & & & $2 \times 4 d f=1$ & $2 \times 4 d f=3$ & & & $2 \times 2$ & $2 \times 4$ & $4 \times 4$ \\
\hline \multicolumn{10}{|c|}{ Empirical $\alpha$} \\
\hline $\mathrm{F}$ & Null & $.0453+$ & .0490 & .0452 & $.0466+$ & Null & .0478 & .0472 & .0494 \\
\hline RO & Null & $.0550+$ & .0550 & .0518 & $.0648+$ & Null & .0594 & .0582 & .0604 \\
\hline $\mathrm{H}$ & Null & $.0540+$ & .0660 & .0552 & $.0855+$ & Null & .0588 & .0606 & .0590 \\
\hline M & Null & $.0644+$ & .0686 & .0594 & $.0890+$ & Null & .0690 & .0644 & .0624 \\
\hline RT & 1 & $.0524+$ & .0496 & .0518 & $.0519+$ & 1 & .0546 & .0522 & .0532 \\
\hline PS & 1 & $.0520+$ & .0482 & .0448 & $.0488+$ & 1 & .0568 & .0440 & .0406 \\
\hline RT & 2 & $.0488+$ & .0518 & .0518 & $.0515+$ & 4 & .0536 & .0554 & .0556 \\
\hline PS & 2 & $.0107+$ & .0168 & .0062 & $.0102+$ & 4 & .0134 & .0090 & .0096 \\
\hline RT & 3 & $.0468+$ & .0516 & .0492 & $.0527+$ & 7 & .0504 & .0562 & .0574 \\
\hline PS & 3 & $.0041+$ & .0068 & .0026 & $.0043+$ & 7 & .0042 & .0030 & .0028 \\
\hline $\mathrm{RT}$ & $4 \& 10$ & $.0499+$ & .0524 & .0482 & $.0535+$ & 10 & .0542 & .0526 & .0564 \\
\hline PS & $4 \& 10$ & $.0105+$ & .0134 & .0094 & $.0163+$ & 10 & .0128 & .0120 & .0080 \\
\hline RT & $5 \& 11$ & $.0125+$ & .0538 & .0426 & $.0510+$ & 13 & .0138 & .0522 & .0590 \\
\hline PS & $5 \& 11$ & $.0029+$ & .0060 & .0028 & $.0047+$ & 13 & .0038 & .0046 & .0032 \\
\hline RT & $6 \& 12$ & $.0101+$ & .0512 & .0428 & $.0503+$ & 16 & .0112 & .0538 & .0604 \\
\hline PS & $6 \& 12$ & $.0012+$ & .0024 & .0010 & $.0025+$ & 16 & .0024 & .0012 & .0012 \\
\hline RT & $7 \& 19$ & $.0472+$ & .0514 & .0480 & $.0518+$ & 19 & .0512 & .0504 & .0570 \\
\hline PS & $7 \& 19$ & $.0032+$ & .0036 & .0026 & $.0078+$ & 19 & .0044 & .0032 & .0032 \\
\hline
\end{tabular}


TABLE 4 (continued)

\begin{tabular}{|c|c|c|c|c|c|c|c|c|c|}
\hline \multirow[b]{2}{*}{ Stat } & \multirow[b]{2}{*}{ Case } & \multirow[b]{2}{*}{$2 \times 2$} & \multicolumn{2}{|c|}{ Main effect } & \multirow[b]{2}{*}{$4 \times 4$} & \multirow[b]{2}{*}{ Case } & \multicolumn{3}{|c|}{ Interaction } \\
\hline & & & $2 \times 4 d f=1$ & $2 \times 4 d f=3$ & & & $2 \times 2$ & $2 \times 4$ & $4 \times 4$ \\
\hline RT & $8 \& 20$ & $.0092+$ & .0512 & .0394 & $.0512+$ & 22 & .0104 & .0518 & .0600 \\
\hline PS & $8 \& 20$ & $.0010+$ & .0024 & .0014 & $.0027+$ & 22 & .0014 & .0014 & .0016 \\
\hline RT & $9 \& 21$ & $.0034+$ & .0518 & .0374 & $.0502+$ & 25 & .0036 & .0610 & .0656 \\
\hline PS & $9 \& 21$ & $.0003+$ & .0012 & .0002 & $.0014+$ & 25 & .0004 & .0006 & .0008 \\
\hline \multicolumn{10}{|c|}{ Empirical power (medium) } \\
\hline $\mathrm{F}$ & Med. & $.8491+$ & .8524 & .8592 & $.8450+$ & Med. & .8530 & .8486 & .8370 \\
\hline RO & Med. & $.9031+$ & .9328 & .9388 & $.9542+$ & Med. & .9002 & .9436 & .9662 \\
\hline $\mathrm{H}$ & Med. & $.9030+$ & .9472 & .9426 & $.9676+$ & Med. & .8998 & .9474 & .9670 \\
\hline M & Med. & $.9166+$ & .9488 & .9462 & $.9688+$ & Med. & .9116 & .9522 & .9698 \\
\hline RT & $10 \& 4$ & $.9116+$ & .9532 & .9556 & $.9812+$ & 2 & .9072 & .9508 & .9704 \\
\hline PS & $10 \& 4$ & $.9150+$ & .9546 & .9528 & $.9800+$ & 2 & .9126 & .9462 & .9650 \\
\hline RT & $11 \& 5$ & $.8789+$ & .9184 & .9262 & $.9534+$ & 5 & .8766 & .9322 & .9610 \\
\hline PS & $11 \& 5$ & $.7513+$ & .8328 & .7812 & $.8718+$ & 5 & .7446 & .7964 & .8746 \\
\hline RT & $12 \& 6$ & $.8490+$ & .8972 & .9094 & $.9366+$ & 8 & .8482 & .9144 & .9472 \\
\hline PS & $12 \& 6$ & $.5156+$ & .6952 & .5724 & $.7420+$ & 8 & .5076 & .5934 & .7472 \\
\hline RT & 13 & $.8762+$ & .9298 & .9350 & $.9603+$ & 11 & .8802 & .9378 & .9610 \\
\hline PS & 13 & $.7527+$ & .8486 & .8572 & $.9231+$ & 11 & .7600 & .8644 & .8744 \\
\hline RT & 14 & $.6597+$ & .8904 & .9144 & $.9396+$ & 14 & .6576 & .9166 & .9442 \\
\hline PS & 14 & $.4210+$ & .6978 & .6622 & $.7939+$ & 14 & .4148 & .6622 & .7244 \\
\hline
\end{tabular}


TABLE 4 (continued)

\begin{tabular}{|c|c|c|c|c|c|c|c|c|c|}
\hline \multirow[b]{2}{*}{ Stat } & \multirow[b]{2}{*}{ Case } & \multirow[b]{2}{*}{$2 \times 2$} & \multicolumn{2}{|c|}{ Main effect } & \multirow[b]{2}{*}{$4 \times 4$} & \multirow[b]{2}{*}{ Case } & \multicolumn{3}{|c|}{ Interaction } \\
\hline & & & $2 \times 4 d f=1$ & $2 \times 4 d f=3$ & & & $2 \times 2$ & $2 \times 4$ & $4 \times 4$ \\
\hline RT & 15 & $.5045+$ & .8710 & .8968 & $.9213+$ & 17 & .4994 & .9058 & .9342 \\
\hline PS & 15 & $.1716+$ & .5600 & .4702 & $.6375+$ & 17 & .1608 & .5046 & .5830 \\
\hline RT & $16 \& 22$ & $.8488+$ & .9120 & .9180 & $.9456+$ & 20 & .8528 & .9044 & .9440 \\
\hline PS & $16 \& 22$ & $.5055+$ & .7246 & .6922 & $.8465+$ & 20 & .5000 & .6600 & .7414 \\
\hline RT & $17 \& 23$ & $.5087+$ & .8764 & .9016 & $.9298+$ & 23 & .4948 & .8846 & .9348 \\
\hline PS & $17 \& 23$ & $.1759+$ & .5818 & .5274 & $.7045+$ & 23 & .1608 & .4724 & .5844 \\
\hline RT & $18 \& 24$ & $.1882+$ & .8622 & .8798 & $.9108+$ & 26 & .1774 & .8760 & .9282 \\
\hline PS & $18 \& 24$ & $.0424+$ & .4620 & .3578 & $.5548+$ & 26 & .0360 & .3328 & .4550 \\
\hline \multicolumn{10}{|c|}{ Empirical power (large) } \\
\hline $\mathrm{F}$ & Lar. & $.9711+$ & .9782 & .9742 & $.9794+$ & Lar. & .9664 & .9738 & .9806 \\
\hline RO & Lar. & $.9847+$ & .9956 & .9944 & $.9977+$ & Lar. & .9818 & .9936 & .9990 \\
\hline $\mathrm{H}$ & Lar. & $.9846+$ & .9956 & .9948 & $.9988+$ & Lar. & .9818 & .9942 & .9992 \\
\hline M & Lar. & $.9877+$ & .9962 & .9954 & $.9988+$ & Lar. & .9848 & .9954 & .9992 \\
\hline RT & $19 \& 7$ & $.9866+$ & .9972 & .9956 & $.9994+$ & 3 & .9834 & .9948 & .9988 \\
\hline PS & $19 \& 7$ & $.9876+$ & .9966 & .9946 & $.9993+$ & 3 & .9842 & .9944 & .9988 \\
\hline RT & $20 \& 8$ & $.9856+$ & .9950 & .9932 & $.9973+$ & 6 & .9850 & .9952 & .9990 \\
\hline PS & $20 \& 8$ & $.9706+$ & .9872 & .9756 & $.9930+$ & 6 & .9668 & .9782 & .9946 \\
\hline RT & $21 \& 9$ & $.9767+$ & .9906 & .9902 & $.9963+$ & 9 & .9732 & .9924 & .9984 \\
\hline PS & $21 \& 9$ & $.8988+$ & .9626 & .9240 & $.9740+$ & 9 & .8892 & .9412 & .9834 \\
\hline
\end{tabular}


TABLE 4 (continued)

\begin{tabular}{|c|c|c|c|c|c|c|c|c|c|}
\hline \multirow[b]{2}{*}{ Stat } & \multirow[b]{2}{*}{ Case } & \multirow[b]{2}{*}{$2 \times 2$} & \multicolumn{2}{|c|}{ Main effect } & \multirow[b]{2}{*}{$4 \times 4$} & \multirow[b]{2}{*}{ Case } & \multicolumn{3}{|c|}{ Interaction } \\
\hline & & & $2 \times 4 d f=1$ & $2 \times 4 d f=3$ & & & $2 \times 2$ & $2 \times 4$ & $4 \times 4$ \\
\hline RT & $22 \& 16$ & $.9857+$ & .9952 & .9940 & $.9982+$ & 12 & .9828 & .9952 & .9990 \\
\hline PS & $22 \& 16$ & $.9697+$ & .9896 & .9848 & $.9958+$ & 12 & .9666 & .9854 & .9952 \\
\hline RT & $23 \& 17$ & $.9520+$ & .9906 & .9894 & $.9968+$ & 15 & .9452 & .9928 & .9984 \\
\hline PS & $23 \& 17$ & $.8867+$ & .9662 & .9432 & $.9831+$ & 15 & .8826 & .9502 & .9818 \\
\hline RT & $24 \& 18$ & $.9123+$ & .9856 & .9886 & $.9957+$ & 18 & .9076 & .9900 & .9972 \\
\hline PS & $24 \& 18$ & $.7318+$ & .9238 & .8882 & $.9558+$ & 18 & .7160 & .9000 & .9530 \\
\hline RT & 25 & $.9761+$ & .9922 & .9916 & $.9971+$ & 21 & .9732 & .9926 & .9984 \\
\hline PS & 25 & $.8959+$ & .9696 & .9582 & $.9896+$ & 21 & .8956 & .9628 & .9826 \\
\hline RT & 26 & $.9138+$ & .9884 & .9884 & $.9953+$ & 24 & .9080 & .9886 & .9976 \\
\hline PS & 26 & $.7261+$ & .9406 & .8974 & $.9672+$ & 24 & .7228 & .9010 & .9528 \\
\hline RT & 27 & $.7377+$ & .9836 & .9874 & $.9947+$ & 27 & .7302 & .9878 & .9964 \\
\hline PS & 27 & $.4591+$ & .8814 & .8210 & $.9323+$ & 27 & .4518 & .8284 & .8994 \\
\hline
\end{tabular}

+ Entry is averaged over two main effects 
TABLE 5

Exponential distribution, $\mathrm{n}=10$

\begin{tabular}{|c|c|c|c|c|c|c|c|c|c|}
\hline \multirow[b]{2}{*}{ Stat } & \multirow[b]{2}{*}{ Case } & \multirow[b]{2}{*}{$2 \times 2$} & \multicolumn{2}{|c|}{ Main effect } & \multirow[b]{2}{*}{$4 \times 4$} & \multirow[b]{2}{*}{ Case } & \multicolumn{3}{|c|}{ Interaction } \\
\hline & & & $2 \times 4 d f=1$ & $2 \times 4 d f=3$ & & & $2 \times 2$ & $2 \times 4$ & $4 \times 4$ \\
\hline \multicolumn{10}{|c|}{ Empirical $\alpha$} \\
\hline $\mathrm{F}$ & Null & $.0440+$ & .0456 & .0434 & $.0504+$ & Null & .0518 & .0506 & .0444 \\
\hline RO & Null & $.0478+$ & .0466 & .0514 & $.0572+$ & Null & .0570 & .0562 & .0504 \\
\hline $\mathrm{H}$ & Null & $.0463+$ & .0498 & .0512 & $.0655+$ & Null & .0558 & .0558 & .0482 \\
\hline M & Null & $.0507+$ & .0508 & .0538 & $.0664+$ & Null & .0580 & .0594 & .0500 \\
\hline RT & 1 & $.0465+$ & .0444 & .0480 & $.0524+$ & 1 & .0578 & .0494 & .0434 \\
\hline PS & 1 & $.0450+$ & .0430 & .0454 & $.0508+$ & 1 & .0556 & .0472 & .0394 \\
\hline $\mathrm{RT}$ & 2 & $.0482+$ & .0470 & .0496 & $.0525+$ & 4 & .0576 & .0542 & .0474 \\
\hline PS & 2 & $.0194+$ & .0220 & .0144 & $.0227+$ & 4 & .0202 & .0196 & .0222 \\
\hline RT & 3 & $.0461+$ & .0480 & .0486 & $.0505+$ & 7 & .0548 & .0532 & .0488 \\
\hline PS & 3 & $.0102+$ & .0150 & .0072 & $.0132+$ & 7 & .0096 & .0128 & .0104 \\
\hline RT & $4 \& 10$ & $.0469+$ & .0486 & .0490 & $.0521+$ & 10 & .0552 & .0536 & .0502 \\
\hline PS & $4 \& 10$ & $.0173+$ & .0238 & .0218 & $.0307+$ & 10 & .0210 & .0228 & .0218 \\
\hline RT & $5 \& 11$ & $.0330+$ & .0494 & .0480 & $.0501+$ & 13 & .0384 & .0558 & .0534 \\
\hline PS & $5 \& 11$ & $.0086+$ & .0132 & .0078 & $.0150+$ & 13 & .0098 & .0120 & .0130 \\
\hline RT & $6 \& 12$ & $.0286+$ & .0494 & .0488 & $.0491+$ & 16 & .0302 & .0592 & .0548 \\
\hline PS & $6 \& 12$ & $.0041+$ & .0102 & .0042 & $.0090+$ & 16 & .0048 & .0076 & .0068 \\
\hline $\mathrm{RT}$ & $7 \& 19$ & $.0465+$ & .0486 & .0498 & $.0525+$ & 19 & .0526 & .0510 & .0496 \\
\hline PS & $7 \& 19$ & $.0086+$ & .0166 & .0050 & $.0194+$ & 19 & .0092 & .0074 & .0122 \\
\hline
\end{tabular}


TABLE 5 (continued)

\begin{tabular}{|c|c|c|c|c|c|c|c|c|c|}
\hline \multirow[b]{2}{*}{ Stat } & \multirow[b]{2}{*}{ Case } & \multirow[b]{2}{*}{$2 \times 2$} & \multicolumn{2}{|c|}{ Main effect } & \multirow[b]{2}{*}{$4 \times 4$} & \multirow[b]{2}{*}{ Case } & \multicolumn{3}{|c|}{ Interaction } \\
\hline & & & $2 \times 4 d f=1$ & $2 \times 4 d f=3$ & & & $2 \times 2$ & $2 \times 4$ & $4 \times 4$ \\
\hline RT & $8 \& 20$ & $.0285+$ & .0468 & .0460 & $.0506+$ & 22 & .0308 & .0518 & .0562 \\
\hline PS & $8 \& 20$ & $.0044+$ & .0094 & .0028 & $.0102+$ & 22 & .0040 & .0038 & .0068 \\
\hline RT & $9 \& 21$ & $.0200+$ & .0476 & .0450 & $.0502+$ & 25 & .0178 & .0612 & .0588 \\
\hline PS & $9 \& 21$ & $.0028+$ & .0080 & .0022 & $.0063+$ & 25 & .0024 & .0032 & .0036 \\
\hline \multicolumn{10}{|c|}{ Empirical power (medium) } \\
\hline $\mathrm{F}$ & Med. & $.8482+$ & .8554 & .8544 & $.8223+$ & Med. & .8428 & .8464 & .8528 \\
\hline RO & Med. & $.9486+$ & .9652 & .9698 & $.9698+$ & Med. & .9394 & .9694 & .9878 \\
\hline $\mathbf{H}$ & Med. & $.9479+$ & .9684 & .9716 & $.9741+$ & Med. & .9390 & .9698 & .9878 \\
\hline $\mathbf{M}$ & Med. & $.9512+$ & .9692 & .9730 & $.9748+$ & Med. & .9424 & .9712 & .9880 \\
\hline RT & 10 & $.9570+$ & .9792 & .9798 & $.9869+$ & 2 & .9486 & .9742 & .9902 \\
\hline PS & 10 & $.9551+$ & .9802 & .9796 & $.9868+$ & 2 & .9474 & .9732 & .9898 \\
\hline RT & 11 & $.9303+$ & .9568 & .9596 & $.9687+$ & 5 & .9226 & .9612 & .9834 \\
\hline PS & 11 & $.8814+$ & .9364 & .9164 & $.9413+$ & 5 & .8758 & .9212 & .9662 \\
\hline RT & 12 & $.9042+$ & .9444 & .9464 & $.9551+$ & 8 & .9008 & .9466 & .9756 \\
\hline PS & 12 & $.7666+$ & .8798 & .8284 & $.8824+$ & 8 & .7592 & .8418 & .9328 \\
\hline RT & 13 & $.9302+$ & .9620 & .9652 & $.9758+$ & 11 & .9206 & .9640 & .9846 \\
\hline PS & 13 & $.8791+$ & .9396 & .9410 & $.9622+$ & 11 & .8678 & .9378 & .9650 \\
\hline RT & 14 & $.8257+$ & .9366 & .9540 & $.9591+$ & 14 & .8176 & .9452 & .9750 \\
\hline PS & 14 & $.6955+$ & .8806 & .8756 & $.9064+$ & 14 & .6836 & .8668 & .9326 \\
\hline
\end{tabular}


TABLE 5 (continued)

\begin{tabular}{|c|c|c|c|c|c|c|c|c|c|}
\hline \multirow[b]{2}{*}{ Stat } & \multirow[b]{2}{*}{ Case } & \multirow[b]{2}{*}{$2 \times 2$} & \multicolumn{2}{|c|}{ Main effect } & \multirow[b]{2}{*}{$4 \times 4$} & \multirow[b]{2}{*}{ Case } & \multicolumn{3}{|c|}{ Interaction } \\
\hline & & & $2 \times 4 d f=1$ & $2 \times 4 d f=3$ & & & $2 \times 2$ & $2 \times 4$ & $4 \times 4$ \\
\hline RT & 15 & $.7545+$ & .9210 & .9406 & $.9444+$ & 17 & .7472 & .9384 & .9688 \\
\hline PS & 15 & $.4809+$ & .8160 & .7744 & $.8390+$ & 17 & .4738 & .7992 & .8824 \\
\hline RT & 16 & $.9037+$ & .9468 & .9460 & $.9644+$ & 20 & .8942 & .9378 & .9764 \\
\hline PS & 16 & $.7629+$ & .8916 & .8180 & $.9298+$ & 20 & .7582 & .8110 & .9294 \\
\hline RT & 17 & $.7499+$ & .9270 & .9368 & $.9495+$ & 23 & .7396 & .9232 & .9688 \\
\hline PS & 17 & $.4847+$ & .8256 & .7360 & $.8609+$ & 23 & .4808 & .7184 & .8810 \\
\hline RT & 18 & $.5818+$ & .9144 & .9216 & $.9352+$ & 26 & .5818 & .9154 & .9628 \\
\hline PS & 18 & $.2382+$ & .7600 & .6366 & $.7896+$ & 26 & .2418 & .6416 & .8256 \\
\hline \multicolumn{10}{|c|}{ Empirical power (large) } \\
\hline $\mathrm{F}$ & Lar. & $.9766+$ & .9974 & .9816 & $.9834+$ & Lar. & .9740 & .9846 & .9858 \\
\hline RO & Lar. & $.9961+$ & 1.0000 & .9994 & $.9995+$ & Lar. & .9946 & .9992 & .9998 \\
\hline $\mathrm{H}$ & Lar. & $.9959+$ & 1.0000 & .9994 & $.9997+$ & Lar. & .9946 & .9992 & .9998 \\
\hline $\mathbf{M}$ & Lar. & $.9964+$ & 1.0000 & .9994 & $.9997+$ & Lar. & .9950 & .9992 & .9998 \\
\hline RT & 19 & $.9963+$ & 1.0000 & .9996 & $.9999+$ & 3 & .9960 & .9990 & .9996 \\
\hline PS & 19 & $.9965+$ & 1.0000 & .9996 & $.9999+$ & 3 & .9956 & .9990 & .9996 \\
\hline RT & 20 & $.9961+$ & 1.0000 & .9990 & $.9996+$ & 6 & .9952 & .9982 & .9998 \\
\hline PS & 20 & $.9925+$ & 1.0000 & .9966 & $.9985+$ & 6 & .9910 & .9972 & .9994 \\
\hline RT & 21 & $.9933+$ & 1.0000 & .9976 & $.9992+$ & 9 & .9920 & .9978 & .9998 \\
\hline PS & 21 & $.9785+$ & .9998 & .9882 & $.9964+$ & 9 & .9734 & .9922 & .9988 \\
\hline
\end{tabular}


TABLE 5 (continued)

\begin{tabular}{|c|c|c|c|c|c|c|c|c|c|}
\hline \multirow[b]{2}{*}{ Stat } & \multirow[b]{2}{*}{ Case } & \multirow[b]{2}{*}{$2 \times 2$} & \multicolumn{2}{|c|}{ Main effect } & \multirow[b]{2}{*}{$4 \times 4$} & \multirow[b]{2}{*}{ Case } & \multicolumn{3}{|c|}{ Interaction } \\
\hline & & & $2 \times 4 d f=1$ & $2 \times 4 d f=3$ & & & $2 \times 2$ & $2 \times 4$ & $4 \times 4$ \\
\hline RT & 22 & $.9950+$ & 1.0000 & .9990 & $.9994+$ & 12 & .9952 & .9988 & .9998 \\
\hline PS & 22 & $.9914+$ & 1.0000 & .9978 & $.9988+$ & 12 & .9906 & .9978 & .9994 \\
\hline RT & 23 & $.9869+$ & 1.0000 & .9972 & $.9992+$ & 15 & .9842 & .9980 & .9996 \\
\hline PS & 23 & $.9709+$ & .9992 & .9920 & $.9965+$ & 15 & .9684 & .9928 & .9984 \\
\hline RT & 24 & $.9719+$ & .9998 & .9964 & $.9980+$ & 18 & .9698 & .9966 & .9996 \\
\hline PS & 24 & $.9224+$ & .9980 & .9822 & $.9940+$ & 18 & .9200 & .9848 & .9968 \\
\hline RT & 25 & $.9927+$ & 1.0000 & .9974 & $.9986+$ & 21 & .9924 & .9980 & .9996 \\
\hline PS & 25 & $.9773+$ & .9996 & .9880 & $.9976+$ & 21 & .9748 & .9872 & .9988 \\
\hline RT & 26 & $.9730+$ & .9998 & .9962 & $.9979+$ & 24 & .9712 & .9954 & .9996 \\
\hline PS & 26 & $.9239+$ & .9980 & .9766 & $.9946+$ & 24 & .9198 & .9726 & .9962 \\
\hline RT & 27 & $.9326+$ & 1.0000 & .9964 & $.9974+$ & 27 & .9260 & .9942 & .9994 \\
\hline PS & 27 & $.8056+$ & .9968 & .9630 & $.9903+$ & 27 & .8022 & .9536 & .9906 \\
\hline
\end{tabular}

+ Entry is averaged over two main effects. 
TABLE 6

Mixed normal distribution, $\mathrm{n}=5$

\begin{tabular}{|c|c|c|c|c|c|c|c|c|c|}
\hline \multirow[b]{2}{*}{ Stat } & \multirow[b]{2}{*}{ Case } & \multirow[b]{2}{*}{$2 \times 2$} & \multicolumn{2}{|c|}{ Main effect } & \multirow[b]{2}{*}{$4 \times 4$} & \multirow[b]{2}{*}{ Case } & \multicolumn{3}{|c|}{ Interaction } \\
\hline & & & $2 \times 4 d f=1$ & $2 \times 4 d f=3$ & & & $2 \times 2$ & $2 \times 4$ & $4 \times 4$ \\
\hline \multicolumn{10}{|c|}{ Empirical $\alpha$} \\
\hline $\mathrm{F}$ & Null & $.0237+$ & .0188 & .0226 & $.0291+$ & Null & .0192 & .0186 & .0410 \\
\hline RO & Null & $.0314+$ & .2486 & .0320 & $.4514+$ & Null & .0282 & .1212 & .1372 \\
\hline $\mathrm{H}$ & Null & $.0293+$ & .2172 & .0200 & $.3537+$ & Null & .0254 & .0434 & .0208 \\
\hline M & Null & $.0345+$ & .2244 & .0228 & $.3595+$ & Null & .0330 & .0482 & .0220 \\
\hline RT & 1 & $.0503+$ & .0532 & .0534 & $.0522+$ & 1 & .0458 & .0486 & .0538 \\
\hline PS & 1 & $.0516+$ & .0494 & .0448 & $.0496+$ & 1 & .0472 & .0438 & .0404 \\
\hline RT & 2 & $.0461+$ & .0482 & .0510 & $.0493+$ & 4 & .0410 & .0360 & .0534 \\
\hline PS & 2 & $.0019+$ & .0004 & .0002 & $.0000+$ & 4 & .0018 & .0002 & .0000 \\
\hline RT & 3 & $.0462+$ & .0482 & .0506 & $.0497+$ & 7 & .0414 & .0328 & .0504 \\
\hline PS & 3 & $.0015+$ & .0004 & .0002 & $.0000+$ & 7 & .0014 & .0000 & .0000 \\
\hline RT & $4 \& 10$ & $.0480+$ & .0410 & .0480 & $.0442+$ & 10 & .0412 & .0438 & .0572 \\
\hline PS & $4 \& 10$ & $.0025+$ & .0002 & .0004 & $.0000+$ & 10 & .0018 & .0010 & .0002 \\
\hline $\mathrm{RT}$ & $5 \& 11$ & $.0024+$ & .0290 & .0316 & $.0336+$ & 13 & .0024 & .2122 & .1512 \\
\hline PS & $5 \& 11$ & $.0013+$ & .0006 & .0006 & $.0000+$ & 13 & .0010 & .0004 & .0008 \\
\hline $\mathrm{RT}$ & $6 \& 12$ & $.0037+$ & .0100 & .0240 & $.0289+$ & 16 & .0036 & .1902 & .1662 \\
\hline PS & $6 \& 12$ & $.0012+$ & .0006 & .0006 & $.0000+$ & 16 & .0012 & .0004 & .0006 \\
\hline RT & $7 \& 19$ & $.0480+$ & .0392 & .0480 & $.0397+$ & 19 & .0412 & .0436 & .0546 \\
\hline PS & $7 \& 19$ & $.0022+$ & .0002 & .0004 & $.0000+$ & 19 & .0012 & .0010 & .0002 \\
\hline
\end{tabular}


TABLE 6 (continued)

\begin{tabular}{|c|c|c|c|c|c|c|c|c|c|}
\hline \multirow[b]{2}{*}{ Stat } & \multirow[b]{2}{*}{ Case } & \multirow[b]{2}{*}{$2 \times 2$} & \multicolumn{2}{|c|}{ Main effect } & \multirow[b]{2}{*}{$4 \times 4$} & \multirow[b]{2}{*}{ Case } & \multicolumn{3}{|c|}{ Interaction } \\
\hline & & & $2 \times 4 d f=1$ & $2 \times 4 d f=3$ & & & $2 \times 2$ & $2 \times 4$ & $4 \times 4$ \\
\hline RT & $8 \& 20$ & $.0030+$ & .0130 & .0316 & $.0351+$ & 22 & .0034 & .1616 & .1704 \\
\hline PS & $8 \& 20$ & $.0012+$ & .0004 & .0006 & $.0000+$ & 22 & .0010 & .0002 & .0004 \\
\hline RT & $9 \& 21$ & $.0030+$ & .0220 & .0328 & $.0299+$ & 25 & .0030 & .3620 & .2450 \\
\hline PS & $9 \& 21$ & $.0010+$ & .0004 & .0006 & $.0000+$ & 25 & .0010 & .0004 & .0004 \\
\hline \multicolumn{10}{|c|}{ Empirical power (medium) } \\
\hline $\mathrm{F}$ & Med. & $.8037+$ & .8184 & .8212 & $.8269+$ & Med. & .8040 & .8244 & .8212 \\
\hline RO & Med. & $.9780+$ & .9924 & .9978 & $.9999+$ & Med. & .9814 & .9984 & 1.0000 \\
\hline $\mathrm{H}$ & Med. & $.9772+$ & .9928 & .9976 & $.9998+$ & Med. & .9800 & .9984 & 1.0000 \\
\hline $\mathbf{M}$ & Med. & $.9789+$ & .9930 & .9976 & $.9998+$ & Med. & .9828 & .9984 & 1.0000 \\
\hline RT & $10 \& 4$ & $.9891+$ & 1.0000 & 1.0000 & $1.0000+$ & 2 & .9904 & 1.0000 & 1.0000 \\
\hline PS & $10 \& 4$ & $.9885+$ & .9998 & 1.0000 & $1.0000+$ & 2 & .9906 & 1.0000 & 1.0000 \\
\hline RT & $11 \& 5$ & $.9434+$ & .9904 & .9982 & $1.0000+$ & 5 & .9466 & .9988 & 1.0000 \\
\hline PS & $11 \& 5$ & $.8814+$ & .9644 & .9780 & $.9994+$ & 5 & .8876 & .9904 & 1.0000 \\
\hline RT & $12 \& 6$ & $.8404+$ & .9806 & .9874 & $.9995+$ & 8 & .8420 & .9936 & 1.0000 \\
\hline PS & $12 \& 6$ & $.2212+$ & .8672 & .8234 & $.9901+$ & 8 & .2176 & .8932 & .9956 \\
\hline $\mathrm{RT}$ & 13 & $.9436+$ & .9986 & .9990 & $1.0000+$ & 11 & .9454 & .9994 & 1.0000 \\
\hline PS & 13 & $.8834+$ & .9926 & .9954 & $.9999+$ & 11 & .8820 & .9970 & 1.0000 \\
\hline RT & 14 & $.6434+$ & .9842 & .9948 & .9999+ & 14 & .6464 & .9952 & 1.0000 \\
\hline PS & 14 & $.4109+$ & .9162 & .9366 & $.9950+$ & 14 & .4150 & .9574 & .9954 \\
\hline
\end{tabular}




\begin{tabular}{|c|c|c|c|c|c|c|c|c|c|}
\hline \multirow[b]{2}{*}{ Stat } & \multirow[b]{2}{*}{ Case } & \multirow[b]{2}{*}{$2 \times 2$} & \multicolumn{2}{|c|}{ Main effect } & \multirow[b]{2}{*}{$4 \times 4$} & \multirow[b]{2}{*}{ Case } & \multicolumn{3}{|c|}{ Interaction } \\
\hline & & & $2 \times 4 d f=1$ & $2 \times 4 d f=3$ & & & $2 \times 2$ & $2 \times 4$ & $4 \times 4$ \\
\hline RT & 15 & $.4561+$ & .9754 & .9858 & $.9979+$ & 17 & .4592 & .9946 & 1.0000 \\
\hline PS & 15 & $.0141+$ & .8384 & .7392 & $.9175+$ & 17 & .0182 & .9426 & .9616 \\
\hline RT & $16 \& 22$ & $.8397+$ & .9920 & .9930 & $1.0000+$ & 20 & .8422 & .9728 & 1.0000 \\
\hline PS & $16 \& 22$ & $.2227+$ & .9498 & .9304 & $.9990+$ & 20 & .2184 & .7620 & .9974 \\
\hline $\mathrm{RT}$ & $17 \& 23$ & $.4519+$ & .9738 & .9906 & $.9993+$ & 23 & .4576 & .9788 & 1.0000 \\
\hline PS & $17 \& 23$ & $.0153+$ & .8322 & .8664 & $.9707+$ & 23 & .0140 & .6876 & .9626 \\
\hline RT & $18 \& 24$ & $.0015+$ & .9626 & .9766 & $.9940+$ & 26 & .0022 & .9778 & .9990 \\
\hline PS & $18 \& 24$ & $.0002+$ & .7152 & .5982 & $.7699+$ & 26 & .0002 & .6348 & .9096 \\
\hline \multicolumn{10}{|c|}{ Empirical power (large) } \\
\hline $\mathrm{F}$ & Lar. & $.9282+$ & .9518 & .9454 & $.9556+$ & Lar. & .9308 & .9462 & .9556 \\
\hline RO & Lar. & $.9916+$ & .9988 & .9996 & $1.0000+$ & Lar. & .9924 & .9998 & 1.0000 \\
\hline $\mathrm{H}$ & Lar. & $.9915+$ & .9990 & .9996 & $1.0000+$ & Lar. & .9922 & .9996 & 1.0000 \\
\hline $\mathbf{M}$ & Lar. & $.9917+$ & .9990 & .9996 & $1.0000+$ & Lar. & .9926 & .9998 & 1.0000 \\
\hline RT & $19 \& 7$ & $.9912+$ & 1.0000 & 1.0000 & $1.0000+$ & 3 & .9926 & 1.0000 & 1.0000 \\
\hline PS & $19 \& 7$ & $.9906+$ & 1.0000 & 1.0000 & $1.0000+$ & 3 & .9926 & 1.0000 & 1.0000 \\
\hline $\mathrm{RT}$ & $20 \& 8$ & $.9936+$ & .9998 & .9998 & $1.0000+$ & 6 & .9952 & 1.0000 & 1.0000 \\
\hline PS & $20 \& 8$ & $.9918+$ & .9996 & .9994 & $1.0000+$ & 6 & .9936 & .9992 & 1.0000 \\
\hline RT & $21 \& 9$ & $.9627+$ & .9926 & .9990 & $1.0000+$ & 9 & .9624 & .9996 & 1.0000 \\
\hline PS & $21 \& 9$ & $.9039+$ & .9674 & .9850 & $1.0000+$ & 9 & .9086 & .9948 & 1.0000 \\
\hline
\end{tabular}


TABLE 6 (continued)

\begin{tabular}{|c|c|c|c|c|c|c|c|c|c|}
\hline \multirow[b]{2}{*}{ Stat } & \multirow[b]{2}{*}{ Case } & \multirow[b]{2}{*}{$2 \times 2$} & \multicolumn{2}{|c|}{ Main effect } & \multirow[b]{2}{*}{$4 \times 4$} & \multirow[b]{2}{*}{ Case } & \multicolumn{3}{|c|}{ Interaction } \\
\hline & & & $2 \times 4 d f=1$ & $2 \times 4 d f=3$ & & & $2 \times 2$ & $2 \times 4$ & $4 \times 4$ \\
\hline RT & $22 \& 16$ & $.9935+$ & .9996 & .9998 & $1.0000+$ & 12 & .9948 & 1.0000 & 1.0000 \\
\hline PS & $22 \& 16$ & $.9913+$ & .9996 & .9996 & $1.0000+$ & 12 & .9936 & .9998 & 1.0000 \\
\hline RT & $23 \& 17$ & $.9931+$ & .9990 & .9970 & $1.0000+$ & 15 & .9948 & .9994 & 1.0000 \\
\hline PS & $23 \& 17$ & $.9920+$ & .9962 & .9734 & $1.0000+$ & 15 & .9948 & .9956 & 1.0000 \\
\hline RT & $24 \& 18$ & $.9609+$ & .9930 & .9976 & $1.0000+$ & 18 & .9632 & .9982 & 1.0000 \\
\hline PS & $24 \& 18$ & $.9015+$ & .9614 & .9714 & $.9991+$ & 18 & .9040 & .9838 & 1.0000 \\
\hline RT & 25 & $.9627+$ & .9988 & .9996 & $1.0000+$ & 21 & .9620 & .9996 & 1.0000 \\
\hline PS & 25 & $.9049+$ & .9974 & .9968 & $1.0000+$ & 21 & .9050 & .9984 & 1.0000 \\
\hline RT & 26 & $.9600+$ & .9990 & .9970 & $1.0000+$ & 24 & .9616 & .9986 & 1.0000 \\
\hline PS & 26 & $.8991+$ & .9946 & .9606 & $.9997+$ & 24 & .9004 & .9870 & 1.0000 \\
\hline RT & 27 & $.6796+$ & .9914 & .9974 & $1.0000+$ & 27 & .6822 & .9976 & 1.0000 \\
\hline PS & 27 & $.4328+$ & .9460 & .9574 & $.9972+$ & 27 & .4344 & .9674 & .9956 \\
\hline
\end{tabular}

+ Entry is averaged over two main effects. 
RO evidenced a similar pattern to $\mathrm{H}$ and $\mathrm{M}$, except that the liberal nature of the tests extended to interaction tests in the $2 \times 4$ and $4 \times 4$ designs. PS showed the same pattern that occurred for the normal distribution: conservatism in the presence of other nonnull effects which worsened as the number of nonnull effects or degree of nonnullity increased. RT usually showed conservatism (which was worse for the $2 \times 2$ design) only if both other effects were nonnull, except for liberal interaction tests for the $2 \times 4$ and 4 $\times 4$ designs if both main effects were nonnull. Power results show $F$ to be conservative by as much as $18 \%$ for medium power for the $2 \times 2$ design as compared to RO. However, RO, H, M, and RT all have some problem with liberal $\alpha$, invalidating many power comparisons for all but the $2 \times 2$ design. Additionally, in the $2 \times 2$ design, RT suffers from a power decrement in the presence of both other effects being nonnull. PS is conservative in the presence of other nonnull effects.

For $n=10$ and empirical $\alpha$ (see Table 7), results are very similar to those for $n=5$. The liberal patterns for $\mathrm{RO}, \mathrm{H}$, and $\mathrm{M}$ are not as severe, and $\alpha$ for $F$ seems to become less conservative as the size of the design increases. Power results mirror those for $n=5$.

\section{Conclusions}

Researchers desiring an alternative to the parametric fixed effects twoway ANOVA will find little consolation from the results of this simulation study. While the PS, $\mathrm{H}$, and $\mathrm{M}$ tests all share admirable properties in the theory of their development, they also share, along with RT, some serious problems. PS shows conservatism of both $\alpha$ and power in the presence of other nonnull effects which worsen as the number of nonnull effects or degree of nonnullity increases, consistent with the dampening effect found by Toothaker and Chang (1980) and Lemmer (1980). This lack of independence of the tests makes PS a poor choice. $\mathrm{H}$ has liberal $\alpha$ for some designs and distributions, notably for two cases: small $n$ with normal or exponential distributions and the larger designs when samples are taken from a mixed normal distribution. $\mathrm{M}$ shares many of the problems of $\mathrm{H}$, with even more liberal results for small $n$. RO, in spite of its intuitive appeal, also shares many of the problems of $\mathrm{H}$. Although RO is not as liberal for small $n$ from normal or exponential distributions, it is more liberal in some designs when samples were taken from the mixed normal distribution. RT shares many of the problems of PS, although not to the same degree. Even though the rank tests have asymptotic distributions with known formulas for degrees of freedom, perhaps the small-sample distributions of the statistics would be better fit by $\chi^{2}$ or $F$ distributions with different (adjusted) degrees of freedom. In contrast, $F$ suffers from conservatism with the mixed normal distribution, but it never exhibits a liberal $\alpha$. 
TABLE 7

Mixed normal distribution, $\mathrm{n}=10$

\begin{tabular}{|c|c|c|c|c|c|c|c|c|c|}
\hline \multirow[b]{2}{*}{ Stat } & \multirow[b]{2}{*}{ Case } & \multirow[b]{2}{*}{$2 \times 2$} & \multicolumn{2}{|c|}{ Main effect } & \multirow[b]{2}{*}{$4 \times 4$} & \multirow[b]{2}{*}{ Case } & \multicolumn{3}{|c|}{ Interaction } \\
\hline & & & $2 \times 4 d f=1$ & $2 \times 4 d f=3$ & & & $2 \times 2$ & $2 \times 4$ & $4 \times 4$ \\
\hline \multicolumn{10}{|c|}{ Empirical $\alpha$} \\
\hline $\mathrm{F}$ & Null & $.0177+$ & .0330 & .0294 & $.0406+$ & Null & .0156 & .0238 & .0372 \\
\hline RO & Null & $.0351+$ & .1532 & .0394 & $.2529+$ & Null & .0368 & .0882 & .1030 \\
\hline $\mathrm{H}$ & Null & $.0200+$ & .1036 & .0150 & $.1470+$ & Null & .0244 & .0268 & .0186 \\
\hline M & Null & $.0218+$ & .1056 & .0158 & $.1484+$ & Null & .0260 & .0288 & .0186 \\
\hline RT & 1 & $.0504+$ & .0460 & .0478 & $.0492+$ & 1 & .0532 & .0504 & .0514 \\
\hline PS & 1 & $.0480+$ & .0472 & .0460 & $.0483+$ & 1 & .0516 & .0472 & .0458 \\
\hline RT & 2 & $.0465+$ & .0498 & .0504 & $.0518+$ & 4 & .0492 & .0486 & .0500 \\
\hline PS & 2 & $.0018+$ & .0024 & .0004 & $.0000+$ & 4 & .0020 & .0002 & .0002 \\
\hline RT & 3 & $.0468+$ & .0496 & .0506 & $.0510+$ & 7 & .0494 & .0450 & .0524 \\
\hline PS & 3 & $.0016+$ & .0022 & .0004 & $.0000+$ & 7 & .0020 & .0002 & .0000 \\
\hline RT & $4 \& 10$ & $.0477+$ & .0462 & .0458 & $.0503+$ & 10 & .0518 & .0452 & .0518 \\
\hline PS & $4 \& 10$ & $.0017+$ & .0006 & .0000 & $.0007+$ & 10 & .0018 & .0002 & .0004 \\
\hline RT & $5 \& 11$ & $.0036+$ & .0452 & .0294 & $.0499+$ & 13 & .0028 & .1740 & .1638 \\
\hline PS & $5 \& 11$ & $.0009+$ & .0004 & .0006 & $.0000+$ & 13 & .0008 & .0006 & .0004 \\
\hline RT & $6 \& 12$ & $.0077+$ & .0308 & .0286 & $.0486+$ & 16 & .0078 & .1778 & .1838 \\
\hline PS & $6 \& 12$ & $.0009+$ & .0004 & .0004 & $.0000+$ & 16 & .0008 & .0006 & .0004 \\
\hline RT & $7 \& 19$ & $.0473+$ & .0454 & .0470 & $.0495+$ & 19 & .0514 & .0456 & .0498 \\
\hline PS & $7 \& 19$ & $.0015+$ & .0002 & .0000 & $.0000+$ & 19 & .0018 & .0002 & .0002 \\
\hline
\end{tabular}


TABLE 7 (continued)

\begin{tabular}{|c|c|c|c|c|c|c|c|c|c|}
\hline \multirow[b]{2}{*}{ Stat } & \multirow[b]{2}{*}{ Case } & \multirow[b]{2}{*}{$2 \times 2$} & \multicolumn{2}{|c|}{ Main effect } & \multirow[b]{2}{*}{$4 \times 4$} & \multirow[b]{2}{*}{ Case } & \multicolumn{3}{|c|}{ Interaction } \\
\hline & & & $2 \times 4 d f=1$ & $2 \times 4 d f=3$ & & & $2 \times 2$ & $2 \times 4$ & $4 \times 4$ \\
\hline RT & $8 \& 20$ & $.0088+$ & .0290 & .0284 & $.0482+$ & 22 & .0056 & .0616 & .1840 \\
\hline PS & $8 \& 20$ & $.0009+$ & .0000 & .0004 & $.0000+$ & 22 & .0008 & .0002 & .0006 \\
\hline RT & $9 \& 21$ & $.0044+$ & .0394 & .0322 & $.0469+$ & 25 & .0028 & .2948 & .2888 \\
\hline PS & $9 \& 21$ & $.0009+$ & .0000 & .0002 & $.0000+$ & 25 & .0008 & .0004 & .0006 \\
\hline \multicolumn{10}{|c|}{ Empirical power (medium) } \\
\hline $\mathrm{F}$ & Med. & $.8228+$ & .8308 & .8228 & $.8103+$ & Med. & .8144 & .8336 & .8362 \\
\hline RO & Med. & $.9982+$ & .9992 & 1.0000 & $1.0000+$ & Med. & .9974 & 1.0000 & 1.0000 \\
\hline $\mathrm{H}$ & Med. & $.9981+$ & .9990 & 1.0000 & $1.0000+$ & Med. & .9972 & 1.0000 & 1.0000 \\
\hline M & Med. & $.9982+$ & 9990 & 1.0000 & $1.0000+$ & Med. & .9974 & 1.0000 & 1.0000 \\
\hline RT & $10 \& 4$ & $1.0000+$ & 1.0000 & 1.0000 & $1.0000+$ & 2 & 1.0000 & 1.0000 & 1.0000 \\
\hline PS & $10 \& 4$ & $1.0000+$ & 1.0000 & 1.0000 & $1.0000+$ & 2 & 1.0000 & 1.0000 & 1.0000 \\
\hline RT & $11 \& 5$ & $.9985+$ & 1.0000 & 1.0000 & $1.0000+$ & 5 & .9990 & 1.0000 & 1.0000 \\
\hline PS & $11 \& 5$ & $.9960+$ & 1.0000 & .9998 & $1.0000+$ & 5 & .9964 & 1.0000 & 1.0000 \\
\hline RT & $12 \& 6$ & $.9848+$ & .9998 & .9998 & $1.0000+$ & 8 & .9842 & 1.0000 & 1.0000 \\
\hline PS & $12 \& 6$ & $.9125+$ & .9984 & .9974 & $1.0000+$ & 8 & .9120 & .9998 & 1.0000 \\
\hline RT & 13 & $.9980+$ & 1.0000 & 1.0000 & $1.0000+$ & 11 & .9990 & 1.0000 & 1.0000 \\
\hline PS & 13 & $.9959+$ & 1.0000 & 1.0000 & $1.0000+$ & 11 & .9956 & 1.0000 & 1.0000 \\
\hline RT & 14 & $.8913+$ & .9998 & 1.0000 & $1.0000+$ & 14 & .8910 & 1.0000 & 1.0000 \\
\hline PS & 14 & $.7655+$ & .9990 & 1.0000 & $1.0000+$ & 14 & .7708 & .9998 & 1.0000 \\
\hline
\end{tabular}


TABLE 7 (continued)

\begin{tabular}{|c|c|c|c|c|c|c|c|c|c|}
\hline \multirow[b]{2}{*}{ Stat } & \multirow[b]{2}{*}{ Case } & \multirow[b]{2}{*}{$2 \times 2$} & \multicolumn{2}{|c|}{ Main effect } & \multirow[b]{2}{*}{$4 \times 4$} & \multirow[b]{2}{*}{ Case } & \multicolumn{3}{|c|}{ Interaction } \\
\hline & & & $2 \times 4 d f=1$ & $2 \times 4 d f=3$ & & & $2 \times 2$ & $2 \times 4$ & $4 \times 4$ \\
\hline $\mathrm{RT}$ & 15 & $.6697+$ & .9992 & 1.0000 & $1.0000+$ & 17 & .6718 & 1.0000 & 1.0000 \\
\hline PS & 15 & $.1512+$ & .9910 & .9944 & $.9995+$ & 17 & .1564 & .9994 & 1.0000 \\
\hline $\mathrm{RT}$ & $16 \& 22$ & $.9860+$ & 1.0000 & 1.0000 & $1.0000+$ & 20 & .9832 & .9988 & 1.0000 \\
\hline PS & $16 \& 22$ & $.9121+$ & .9998 & .9974 & $1.0000+$ & 20 & .9096 & .9864 & 1.0000 \\
\hline RT & $17 \& 23$ & $.6687+$ & .9998 & 1.0000 & $1.000+$ & 23 & .6804 & .9990 & 1.0000 \\
\hline PS & $17 \& 23$ & $.1516+$ & .9932 & .9916 & $.9999+$ & 23 & .1610 & .9750 & 1.0000 \\
\hline RT & $18 \& 24$ & $.0190+$ & .9978 & .9994 & $1.0000+$ & 26 & .0218 & .9990 & 1.0000 \\
\hline PS & $18 \& 24$ & $.0006+$ & .9732 & .9638 & $.9983+$ & 26 & .0006 & .9582 & 1.0000 \\
\hline \multicolumn{10}{|c|}{ Empirical power (large) } \\
\hline $\mathrm{F}$ & Lar. & $.9515+$ & .9898 & .9624 & $.9687+$ & Lar. & .9516 & .9642 & .9740 \\
\hline RO & Lar. & $.9998+$ & 1.0000 & 1.0000 & $1.0000+$ & Lar. & .9998 & 1.0000 & 1.0000 \\
\hline $\mathrm{H}$ & Lar. & $.9998+$ & 1.0000 & 1.0000 & $1.0000+$ & Lar. & .9998 & 1.0000 & 1.0000 \\
\hline $\mathbf{M}$ & Lar. & $.9998+$ & 1.0000 & 1.0000 & $1.0000+$ & Lar. & .9998 & 1.0000 & 1.0000 \\
\hline $\mathrm{RT}$ & $19 \& 7$ & $1.0000+$ & 1.0000 & 1.0000 & $1.0000+$ & 3 & 1.0000 & 1.0000 & 1.0000 \\
\hline PS & $19 \& 7$ & $1.0000+$ & 1.0000 & 1.0000 & $1.0000+$ & 3 & 1.0000 & 1.0000 & 1.0000 \\
\hline RT & $20 \& 8$ & $1.0000+$ & 1.0000 & 1.0000 & $1.0000+$ & 6 & 1.0000 & 1.0000 & 1.0000 \\
\hline PS & $20 \& 8$ & $1.0000+$ & 1.0000 & 1.0000 & $1.0000+$ & 6 & 1.0000 & 1.0000 & 1.0000 \\
\hline RT & $21 \& 9$ & $.9991+$ & 1.0000 & 1.0000 & $1.0000+$ & 9 & .9992 & 1.0000 & 1.0000 \\
\hline PS & $21 \& 9$ & $.9971+$ & 1.0000 & 1.0000 & $1.0000+$ & 9 & .9972 & 1.0000 & 1.0000 \\
\hline
\end{tabular}


TABLE 7 (continued)

\begin{tabular}{|c|c|c|c|c|c|c|c|c|c|}
\hline \multirow[b]{2}{*}{ Stat } & \multirow[b]{2}{*}{ Case } & \multirow[b]{2}{*}{$2 \times 2$} & \multicolumn{2}{|c|}{ Main effect } & \multirow[b]{2}{*}{$4 \times 4$} & \multirow[b]{2}{*}{ Case } & \multicolumn{3}{|c|}{ Interaction } \\
\hline & & & $2 \times 4 d f=1$ & $2 \times 4 d f=3$ & & & $2 \times 2$ & $2 \times 4$ & $4 \times 4$ \\
\hline RT & $22 \& 16$ & $1.0000+$ & 1.0000 & 1.0000 & $1.0000+$ & 12 & 1.0000 & 1.0000 & 1.0000 \\
\hline PS & $22 \& 16$ & $1.0000+$ & 1.0000 & 1.0000 & $1.0000+$ & 12 & 1.0000 & 1.0000 & 1.0000 \\
\hline RT & $23 \& 17$ & $1.0000+$ & 1.0000 & 1.0000 & $1.0000+$ & 15 & 1.0000 & 1.0000 & 1.0000 \\
\hline PS & $23 \& 17$ & $1.0000+$ & 1.0000 & 1.0000 & $1.0000+$ & 15 & 1.0000 & 1.0000 & 1.0000 \\
\hline RT & $24 \& 18$ & $.9981+$ & 1.0000 & 1.0000 & $1.0000+$ & 18 & .9978 & 1.0000 & 1.0000 \\
\hline PS & $24 \& 18$ & $.9939+$ & 1.0000 & 1.0000 & $1.0000+$ & 18 & .9930 & 1.0000 & 1.0000 \\
\hline RT & 25 & $.9990+$ & 1.0000 & 1.0000 & $1.0000+$ & 21 & .9994 & 1.0000 & 1.0000 \\
\hline PS & 25 & $.9967+$ & 1.0000 & 1.0000 & $1.0000+$ & 21 & .9968 & .9998 & 1.0000 \\
\hline RT & 26 & $.9979+$ & 1.0000 & 1.0000 & $1.0000+$ & 24 & .9970 & 1.0000 & 1.0000 \\
\hline PS & 26 & $.9925+$ & 1.0000 & 1.0000 & $1.0000+$ & 24 & .9938 & 1.0000 & 1.0000 \\
\hline RT & 27 & $.9073+$ & 1.0000 & 1.0000 & $1.0000+$ & 27 & .9082 & 1.0000 & 1.0000 \\
\hline PS & 27 & $.7819+$ & 1.0000 & 1.0000 & $1.0000+$ & 27 & .7888 & .9994 & 1.0000 \\
\hline
\end{tabular}

+ Entry is averaged over two main effects. 
If a researcher has some knowledge of the type of distribution for the population sampled, a choice may be made from among $F, \mathrm{RO}$, and $\mathrm{H}$, if the cell size is not too small and if the distribution is similar to the normal or exponential distribution. If the distribution is suspected to be like the mixed normal, only the $F$ can be recommended unless the design is $2 \times 2$, in which case RO, $\mathrm{H}$, and $\mathrm{M}$ all have adequate control of $\alpha$ and give higher power than $F$.

Certainly more research is needed for these procedures when the equal variance assumption has been violated, especially in combination with nonnormal distributions of the type often encountered in the real world by researchers. Also, for the rank tests, more work is needed in the following areas: using estimates other than the mean in forming appropriate quantities for aligning and using simple main effect analysis that relies on the qualities of ranking in a one-way design. Finally, more research is needed comparing other transformations on the data (e.g., power transformations) to ranking and use of robust estimators in two-way analyses.

\section{References}

Akritas, M. G. (1990). The rank transform method in some two-factor designs. Journal of the American Statistical Association, 85, 73-78.

Blair, R. C., Sawilowsky, S. S., \& Higgins, J. J. (1987). Limitations of the rank transform statistic in tests for interactions. Communications in Statistics: Simulation and Computation, B16, 1133-1145.

Box, G. E. P., \& Muller, M. E. (1958). A note on the generation of random normal deviates. Annals of Mathematical Statistics, 29, 610-611.

Chen, E. H. (1971). Random normal number generator for 32-bit-word computers. Journal of the American Statistical Association, 66, 400-403.

Conover, W. J., \& Iman, R. L. (1976). On some alternative procedures using ranks for the analysis of experimental designs. Communications in Statistics, A5, 1349-1368.

Conover, W. J., \& Iman, R. L. (1981). Rank transformations as a bridge between parametric and nonparametric statistics. The American Statistician, 35(3), 124-129.

Fawcett, R. F., \& Salter, K. C. (1984). A Monte Carlo study of the F test and three tests based on ranks of treatment effects in randomized block designs. Communications in Statistics: Simulation and Computation, 13(2), 213-225.

Fligner, M. A. (1981). Comment. American Statistician, 35(3), 131-132.

Groggel, D. J. (1987). A Monte Carlo study of rank tests for block designs. Communications in Statistics: Simulation and Computation, 16(3), 601-620.

Harwell, M. R. (1991). Completely randomized factorial analysis of variance using ranks. British Journal of Mathematical and Statistical Psychology, 44, 383-401.

Harwell, M. R., \& Serlin, R. C. (1990). A nonparametric test statistic for the general linear model. Journal of Educational Statistics, 14, 351-371.

Hettmansperger, T. P. (1984). Statistical inference based on ranks. New York: Wiley. Hodges, J. L., Jr., \& Lehmann, E. L. (1962). Rank methods for combination of independent experiments in the analysis of variance. Annals of Mathematical Statistics, 33, 482-497. 
Hora, S. C., \& Conover, W. J. (1984). The F statistic in the two-way layout with rank-score transformed data. Journal of the American Statistical Association, 79, 668-673.

Hora, S. C., \& Iman, R. L. (1988). Asymptotic relative efficiencies of the rank transformation procedures in randomized complete block designs. Journal of the American Statistical Association, 83, 462-470.

Iman, R. L. (1974). A power study of a rank transform for the two-way classification model when interaction may be present. Canadian Journal of Statistics, 2, 227-239.

Iman, R. L., \& Conover, W. J. (1976). A comparison of several rank tests for the two-way layout (SAND76-0631). Albuquerque, NM: Sandia Laboratories.

Iman, R. L., Hora, S. C., \& Conover, W. J. (1984). Comparison of asymptotically distribution-free procedures for the analysis of complete blocks. Journal of the American Statistical Association, 79, 674-685.

Koch, G. G. (1969). Some aspects of the statistical analysis of "split-plot" experiments in completely randomized layouts. Journal of the American Statistical Association, 64, 485-505.

Koch, G. G., \& Sen, P. K. (1968). Some aspects of the statistical analysis of the "mixed model." Biometrics, 24, 27-48.

Lehmann, R. S., \& Bailey, D. E. (1968). Digital computing: Fortran IV and its applications in behavioral science. New York: Wiley.

Lemmer, H. H. (1980). Some empirical results on the two-way analysis of variance with ranks. Communications in Statistics: Theories and Methods, A9, 1427-1438.

McSweeney, M. (1967). An empirical study of two proposed nonparametric tests for main effects and interaction (Doctoral dissertation, University of CaliforniaBerkeley, 1968). Dissertation Abstracts International, 28(10), 4005.

Mehra, K. L., \& Sarangi, J. (1967). Asymptotic efficiency of certain rank tests for comparative experiments. The Annals of Mathematical Statistics, 38, 90-107.

Mehra, K. L., \& Sen, P. K. (1969). On a class of conditionally distribution-free tests for interactions in factorial experiments. The Annals of Mathematical Statistics, $40,658-664$.

Micceri, T. (1989). The unicorn, the normal curve, and other improbable creatures. Psychological Bulletin, 105(1), 156-166.

Puri, M. L., \& Sen, P. K. (1985). Nonparametric methods in general linear models. New York: Wiley.

Reinach, S. G. (1965). A nonparametric analysis for a multiway classification with one element per cell. South African Journal of Agricultural Science, 8, 941-960.

SAS Institute. (1990). SAS procedures guide, Version 6 (3rd ed.). Cary, NC: Author. Sawilowsky, S. S. (1985). Robust and power analysis of the $2 \times 2 \times 2$ ANOVA, rank transformation, random normal scores, and expected normal scores transformation tests (Doctoral dissertation, University of South Florida, 1986). Dissertation Abstracts International, 46(10), 3009A.

Sawilowsky, S. S. (1989, April). Rank transform: The bridge is falling down. Paper presented at the Annual Meeting of the American Educational Research Association, San Francisco.

Sawilowsky, S. S. (1990). Nonparametric tests of interaction in experimental design. Review of Educational Research, 60, 91-126. 
Sawilowsky, S. S., Blair, R. C., \& Higgins, J. J. (1989). An investigation of the Type I error and power properties of the rank transformation procedure in factorial ANOVA. Journal of Educational Statistics, 14, 255-267.

Scheirer, C. J., Ray, W. S., \& Hare, N. (1976). The analysis of ranked data derived from completely randomized factorial designs. Biometrics, 32, 429-434.

Sen, P. K. (1968). On a class of aligned rank order tests in two-way layouts. The Annals of Mathematical Statistics, 39, 1115-1124.

Thompson, G. L., \& Ammann, L. P. (1989). Efficiencies of the rank-transform in twoway models with no interaction. Journal of the American Statistical Association, 84(405), 325-330.

Toothaker, L. E., \& Chang, H. (1980). On the analysis of ranked data derived from completely randomized factorial designs. Journal of Educational Statistics, $5,169-176$.

\section{Authors}

LARRY E. TOOTHAKER is David Ross Boyd Professor of Psychology at the University of Oklahoma, Norman, OK 73019. He specializes in robustness of ANOVA, including repeated measures designs, multiple comparison procedures, and nonparametrics.

DE A. NEWMAN is a PhD Candidate, University of Oklahoma, Norman, OK 73019. She specializes in robustness studies, multiple comparisons, and nonparametrics.

Received December 2, 1992

Revision received September 30, 1993

Accepted December 1, 1993 